\title{
TOPOLOGICAL REALIZATIONS OF CHAIN COMPLEXES II. THE RATIONAL CASE
}

\author{
JUSTIN R. SMITH
}

\begin{abstract}
This paper continues the study of the realizability question for chain complexes. We address the following question:

Given a group $\pi$ and a $Q \pi$-projective chain-complex $T$, does there exist a topological space with fundamental group isomorphic to $\pi$ whose equivariant chain complex is $T$ ?

We essentially answer this question in the affirmative in an important special case and develop a purely algebraic obstruction theory for the problem in the general case.
\end{abstract}

Introduction. This question is of interest in connection with the question of what homotopy types of manifolds exist. One can try to construct a manifold by first realizing its chain-complex with the coproduct that makes it into suitable Poincare duality complex-and then try to use surgery theory to build the manifold.

We will call a CW-complex $X$ 0-split if the canonical map $X \rightarrow$ $K\left(\pi_{1}(X)\right)$ splits up to a homotopy. Then the questions mentioned above are resolved fairly completely for 0 -split spaces by developing a theory of "minimal models" for such $\mathrm{CW}$-complexes that generalizes the theory developed by Sullivan in his "Infinitesimal computations in topology"-see [18].

One of the main results of this paper is:

THeOREM (CoRollary 1.6). Let $\pi$ be any group and $(C \oplus \mathbb{Q}, \Delta)$ be any (cocommutative coassociative) DGA-coalgebra with coproduct $\Delta: C \oplus \mathbb{Q} \rightarrow(C \oplus \mathbb{Q}) \otimes(C \oplus \mathbb{Q})$, where $C$ is a 2-connected free $\mathbb{Q} \pi$-chain complex. Then there exists a 0 -split $C W$ complex $X$ with fundamental group $\pi$ such that the chain-complex of the kernel of the canonical map $C(\tilde{X}) \oplus \mathbb{Q} \rightarrow Q_{*}$ is chain-homotopy equivalent to $C$ via a chainhomotopy equivalence that carries the geometric coproduct to a map homotopic to $\Delta$.

Remarks. 1. Note that this implies the solvability of the Steenrod Problem for $\mathbb{Q}$-modules. Here $Q_{*}$ is a projective $\mathbb{Q} \pi$-resolution of $\mathbb{Q}$. 
In [17] the author proved a stable version of this result. In 1981 Hans Baues and Stephen Halperin proved the solvability of the Steenrod Problem for modules of finite $\mathbb{Q}$-rank (private communication to the author).

2. In general there are many possible inequivalent topological realizations for $C$.

3. This result makes essential use of the fact that $C$ is 0 -split and rational. If we attempt to prove the result over $\mathbb{Z} \pi$ rather than $\mathbb{Q} \pi$ we encounter counterexamples-see [4] and [15]. In the nonsplit rational case there are also counterexamples-see $\S 3$ of the present paper.

4. Using the results of C. T. C. Wall in [19] it is possible to find a topological realization for $C$ whose underlying $(\mathrm{CW})$ chain-complex is isomorphic to $C$ after rationalization.

We also show that:

THEOREM. Every 0 -split $C W$ complex has associated with it a projective DGA-coalgebra over $\mathbb{Q} \pi$ called its 0 -split $\mathbb{Q}$-model. Two 0 -split $C W$-complexes are rationally homotopy equivalent if and only if their fundamental groups are isomorphic and their $\mathbb{Q}$-models are isomorphic as DGA-coalgebras.

Remarks. 1. See 2.14 for the definition of rational homotopy equivalence used here. Essentially two $\mathrm{CW}$-complexes are rationally homotopy equivalent in this paper if their fundamental groups are isomorphic and their universal covering spaces are equivariantly rationally homotopy equivalent (in the usual sense). See 2.12 and Appendix $\mathrm{C}$ for a proof that the $\mathbb{Q}$-models faithfully represent the homotopy category of DGA-coalgebras.

We are using the term "minimal models" to stand for a faithful algebraic representation of the rational homotopy category of 0 -split $\mathrm{CW}$-complexes. Rationally homotopy equivalent 0 -split spaces map into isomorphic algebraic objects.

2. The approach used in the present paper is different from that of Baues in [1] and Baues and Lemaire in [2]. Baues picked the chainalgebra of the loop space of a CW complex as the fundamental invariant. That invariant contains much of the same homotopy-theoretic information about a space as the invariant studied here.

3. Our approach differs somewhat from that of Triantafillou and Rothenberg (see [13]) in that our main invariant of a space is the $D G A$ coalgebra rather than the DG-Lie algebra. Although Quillen proved that the homotopy theory of DG-Lie algebras is equivalent to that of 
DG-coalgebras (and even in our context) - there doesn't appear to be any simple way to get a theory of minimal models for DG-Lie algebras over $\mathbf{Q} \pi$ when $\pi$ is infinite. When $\pi$ is finite the contribution of homological algebra essentially disappears and Triantafillou and Rothenberg were able to develop a theory of minimal models for DG-Lie algebras.

4. Our theory differs from that of Sullivan in that it uses the original chain complex rather than the dual. This means that our theory is a kind of dual to that of Sullivan. In fact, in the simply-connected case it is precisely that.

In the general case we define:

1. For any group $\pi$, a $\mathbb{Q}$-model $\mathfrak{B}(\pi)$ (see 1.9);

2. For any $\mathbb{Q} \pi$-chain-complex, $C$, equipped with a chain-map $c: C \rightarrow$ $\widetilde{K(\pi, 1)}$, an associated complex $C_{\mathfrak{B}}$ and chain-map $c_{\mathfrak{B}}: C_{\mathfrak{B}} \rightarrow \mathfrak{B}(\pi)$ (see $1.15)$. Here $\widetilde{K(\pi, 1)}$ denotes the rational chain-complex of the universal covering space of a $K(\pi, 1)$.

With these definitions in mind our main result is (1.19):

THEOREM. Let $C$ be a $\mathbb{Q} \pi$-chain complex such that $H_{0}(C)=\mathbb{Q}$, $H_{1}(C)=0$. Let $c_{\mathfrak{B}}: C_{\mathfrak{B}} \rightarrow \mathfrak{B}(\pi)$ the associated chain-complex and map. Then $C$ is topologically realizable if and only if there exists a $\mathbb{Q}$-model $M$ and a DGA-coalgebra homomorphism $f: M \rightarrow M(\mathfrak{B}(\pi))$ such that the following diagram commutes up to a chain-homotopy:

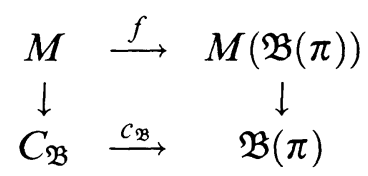

In $\S 3$ of this paper we give an obstruction theory to this happening. It is a purely algebraic version of the obstruction theory presented in [17].

One result in this case is (Proposition 3.3):

THEOREM. Let $\pi$ be a group of $\mathbb{Q}$-homological dimension $\leq 5$ and let $C$ be any projective $\mathbb{Q} \pi$-chain complex. Then there exists at most one obstruction to topologically realizing $C$ : it is $l^{*}(\alpha \otimes v) \in$ $H^{5}\left(C ; H_{2}(C) \otimes I_{2}(\pi)\right)$, where

1. $\alpha \in H^{3}\left(\pi, H_{2}(C)\right)$ is the first homological k-invariant;

2. $l: C_{\mathfrak{B}} \rightarrow \mathfrak{B}(\pi)$ is the projection;

3. $v: \mathfrak{B}(\pi)_{2} \rightarrow I_{2}(\pi)$ maps elements to their homology classes. 
In more generality:

THEOREM. If the $\mathbb{Q}$-homological dimension of $\pi$ is $k>2$ there exist at most $k-4$ significant obstructions to topologically realizing a $\mathbb{Q} \pi$ chain complex.

We also show that the following chain complex is not topologically realizable: $\pi=\mathbf{Z}^{5}$ and $C=Q \oplus_{\alpha} \Sigma^{2} Q$, where $\alpha \in H^{3}(\pi, \mathbb{Q})$ is any nontrivial class, and $Q$ is a projective $\mathbb{Q} \pi$-resolution of $\mathbb{Q}$.

Section 1 describes the connections with Quillen's theory in [11] and develops the topological basis for $\mathbb{Q}$-models.

Section 2 defines $\mathbb{Q}$-models in the 0 -split case and describes their main properties.

Section 3 presents the theory of $\mathbb{Q}$-models in the general case and describes a purely algebraic obstruction theory for realizability of $\mathbb{Q} \pi$ chain-complexes.

Appendix A shows that a certain equivalence of categories developed in $\S 1$ extends to an equivalence of homotopy theories.

Appendix B develops some algebraic background material related to twisted tensor products and DGA-coalgebras.

I am indebted to Sylvain Cappell and Andrew Ranicki for their encouragement. I am also indebted to Drexel University for providing me with a research grant and other support for this work.

1. The initial reduction. In this section we will apply the theory developed by Quillen in [11] to reduce the topological problem of realizing 0 -split rational chain-complexes to a purely algebraic problem.

We begin with:

Definition 1.1. 1. Let 0-Split- $\pi$ denote the category of 0-split CWcomplexes with fundamental group $\pi$, where the morphisms are maps that induce an isomorphism of fundamental groups and commute with splitting maps. A morphism will be called a weak equivalence if it induces isomorphisms in homology of the universal covering spaces.

2 . Let PointedFree- $\pi$ denote the category of pointed simplyconnected $\mathrm{CW}$ complexes that are equipped with a $\pi$-action that is free everywhere except for the basepoint. A morphism will be called a weak equivalence if it induces isomorphisms in homology.

REMARKs. 1. Note that our notion of weak equivalence corresponds to that of homotopy equivalence, due to the fact that the $\pi$-actions are free. 
2. Let $\mathrm{Ho}(0-$ Split- $\pi)$ and $\mathrm{Ho}$ (PointedFree- $\pi)$ denote the corresponding categories localized with respect to weak equivalences.

Definition 1.2. Let the functors $\mathfrak{C}: 0-$ Split- $\pi \rightarrow$ PointedFree- $\pi$, $\mathfrak{F}:$ PointedFree- $\pi \rightarrow 0$-Split- $\pi$ be defined as follows:

If $X \in 0$-Split- $\pi$ with splitting map $s: K(\pi, 1) \rightarrow X$ then:

1. form the universal covering space $\tilde{X}$ with map $\tilde{s}: \widetilde{K(\pi, 1)} \rightarrow$ $\tilde{X}$-here the tildas denote universal covering spaces or induced maps;

2. $\mathfrak{C}(X)$ is the mapping cone of $\tilde{s}$.

If $Y \in$ PointedFree- $\pi$ then

1. form the product $Y \times \overparen{K(\pi, 1)}$, and equip it with the diagonal $\pi$-action;

2. define $\mathfrak{F}(Y)$ to be the quotient $Y \times \overparen{K(\pi, 1)} / \pi$.

Proposition 1.3. The functors $\mathfrak{C}$ and $\mathfrak{F}$ define equivalences of the categories $\mathrm{Ho}(0-$ Split- $\pi)$ and $\mathrm{Ho}($ PointedFree- $\pi)$.

Proof. We need only verify: a. that the functors preserve weak equivalences; b. that $\mathfrak{C} \cdot \mathfrak{F}:$ PointedFree- $\pi \rightarrow$ PointedFree- $\pi$ and $\mathfrak{F}$. $\mathfrak{C}: 0$-Split- $\pi \rightarrow 0-$ Split- $\pi$ induce the identity maps of localized categories (i.e. $\mathfrak{C} \cdot \mathfrak{F}(Y)$ is weakly equivalent to $Y$ and $\mathfrak{F} \cdot \mathfrak{C}(X)$ is weakly equivalent to $X$ ). But both statements are clear.

As in [11, p. 211] we get a sequence of categories:

$\mathfrak{S}_{2}-\pi$-the category of 2-reduced simplicial sets equipped with a $\pi$ action that is free except in dimensions 0 and 1 (each of which have a single element)

$(\mathrm{SGp}-\pi)_{1}$-the category of reduced simplicial groups equipped with a $\pi$-action that is free except at the identity element.

(SCHA- $\pi)_{1}$-the category of reduced simplicial complete Hopf algebras over $\mathbb{Q}$ equipped with a $\pi$-action that is free except in dimension 0 .

$(\text { SLA- } \pi)_{1}$ - the category of reduced simplicial Lie algebras over $\mathbb{Q}$ equipped with a $\pi$-action that is free except in dimension 0 .

$(\mathrm{DGL}-\pi)_{1}$-the category of free reduced differential graded Lie algebras over $\mathbb{Q} \pi{ }^{1}$

\footnotetext{
${ }^{1}$ I.e. the chain-modules are free $\mathbb{Q} \pi$-modules.
} 
$(\mathrm{DGC}-\pi)_{2}$-The category of 2-reduced free differential graded (cocommutative, coassociative) coalgebras over $\mathbb{Q} \pi$. We will call a DGA-coalgebra $C$ 2-reduced if: $C_{0}=\mathbb{Q} ; C_{1}=0$. The copy of $\mathbb{Q}$ in dimension 0 will be called the fixed-point of $C$. Maps between objects of $(\mathrm{DGC}-\pi)_{2}$ are required to preserve fixed points.

As in [11] we get a diagram of pairs of adjoint functors between these categories:

$$
\begin{aligned}
& \text { PointedFree- } \pi \underset{E_{2} \text { Sing }}{\stackrel{\|}{\leftrightarrows}} \mathfrak{S}_{2} \underset{\bar{W}}{\stackrel{G}{\rightleftarrows}}(\mathrm{SGp}-\pi)_{1} \underset{g}{\stackrel{\hat{\mathrm{Q}}}{\rightleftarrows}}(\mathrm{SCHA}-\pi)_{1} \\
& (\mathrm{DGC}-\pi)_{1} \underset{\mathscr{C}}{\stackrel{\mathscr{L}}{\rightleftarrows}} \quad(\mathrm{DGL}-\pi)_{1} \underset{N}{\stackrel{N^{*}}{\rightleftarrows}} \quad \begin{array}{c}
\wp \downarrow \hat{U} \\
(\mathrm{SLA}-\pi)_{1}
\end{array}
\end{aligned}
$$

The functors defined by Quillen are natural with respect (ultimately) to maps of pointed simply-connected spaces so they induce functors between the categories defined in the present paper. As in [11] these functors define equivalences between the categories localized with respect to weak equivalences. Here we make use of the fact that we define weak equivalence with respect to homology rather than homotopy modules. We get the following variation on Theorem I in [11].

THEOREM 1.4. The functors given above induce an equivalence of the localized categories $\mathrm{Ho}(0-\mathrm{Split}-\pi)$ and $\mathrm{Ho}\left((\mathrm{DGC}-\pi)_{2}\right)$.

REMARKs. 1. The proof is essentially the same as that of Theorem I.

2. Equality of objects in the localized categories is defined in the following way:

Two objects $o_{1}$ and $o_{2}$ in a category $C$ are equal in $\mathrm{Ho}(C)$ if and only if there exists a chain of weak equivalences (in $C$ ) $o_{1} \leftrightarrow c_{1} \cdots \leftrightarrow c_{i} \cdots \leftrightarrow o_{2}$. Here $\leftrightarrow$ denotes an arrow going in either direction but not necessarily both directions. A morphism $o_{1} \rightarrow o_{2}$ in $\operatorname{Ho}(C)$ is a morphism $v_{1} \rightarrow v_{2}$, where $v_{i}=o_{i}$ in $\operatorname{Ho}(C), i=1,2$.

3. $\mathrm{Ho}\left((\mathrm{DGC}-\pi)_{2}\right)$ is the homotopy category of free 2-connected cocommutative DGA-coalgebras over $\mathbb{Q} \pi$.

In other words:

COROllary 1.5 Two 0 -split $C W$-complexes $X$ and $Y$ in $\mathrm{Ho}(0-$ Split- $\pi)$ are homotopy equivalent if and only if the commutative 
DGA-coalgebras $\mathscr{C} \circ \wp \circ \hat{\mathbb{Q}} \circ G \circ E_{2} \operatorname{Sing} \circ \mathfrak{C}(X)$ and $\mathscr{C} \circ \wp \circ \hat{\mathbb{Q}} \circ G \circ E_{2} \operatorname{Sing} \circ \mathfrak{C}(Y)$ represent equal objects in $\mathrm{Ho}\left((\mathrm{DGC}-\pi)_{2}\right)$.

COROLlaRY 1.6 Let $\pi$ be any group and $(C \oplus \mathbb{Q}, \Delta)$ be any (commutative coassociative) DGA-coalgebra with coproduct $\Delta: C \oplus \mathbb{Q} \rightarrow(C \oplus$ $\mathbb{Q}) \otimes(C \oplus \mathbb{Q})$, where $C$ is a 2-connected free $\mathbb{Q} \pi$-chain complex. Then there exists a 0 -split $C W$ complex $X$ with fundamental group $\pi$ such that the chain-complex of the kernel of the canonical map $C(\tilde{X}) \otimes \mathbb{Q} \rightarrow \mathbb{Q}_{*}$ is chain-homotopy equivalent to $C$ via a chain-homotopy equivalence that carries the geometric coproduct to a map homotopic to $\Delta$.

REMARKs. 1. Here $\tilde{X}$ represents the universal covering space of $X$, equipped with a free $\pi$-action and the direct summand $\mathbb{Q}$ represents the 0 -dimensional portion of the complex. The chain-complex $C(\tilde{X}) \otimes \mathbb{Q}$ is a free $\mathbb{Q} \pi$-chain-complex and $Q_{*}$ represents a free $\mathbb{Q} \pi$-resolution of $\mathbb{Q}$.

2. The proof of this result follows from consideration of the effect of $\mathfrak{C}$ and $\mathfrak{T}$ on the chain-complex.

Corollary 1.7 If $\pi$ is any group and $C$ is any free 2-connected $\mathbb{Q} \pi$-chain-complex then $C$ is topologically realizable.

REMARK. 1 . Simply equip $C \oplus \mathbb{Q}$ with the trivial coproduct structure that maps $c \in C$ to $1 \otimes c+c \otimes 1$.

2. Note that this implies the solvability of the Steenrod Problem for $\mathbb{Q} \pi$-modules. In [17] the author proved a stable version of this result. In 1981 Hans Baues and Stephen Halperin proved the solvability of the Steenrod Problem for modules of finite Q-rank (private communication to the author).

In [11] Quillen proved that this theory gave an equivalence of homotopy theories between pointed 2-connected topological spaces and $(\mathrm{DGC})_{2}$. Here a homotopy theory is defined in the sense of [12]-a closed model category localized with respect to weak equivalences. Fibrations of spaces corresponded (under the equivalence of homotopy theories mentioned above) to fibrations in $(\mathrm{DGC})_{2}$. These turned out to be twisted tensor products equipped with the untwisted coproduct structure where the fiber is the universal enveloping algebra of a DG Lie algebra.

We prove a similar result.

THEOREM 1.8 The equivalence of the categories $\mathrm{Ho}(0-\mathrm{Split}-\pi)$ and $\mathrm{Ho}\left((\mathrm{DGC}-\pi)_{2}\right)$ induces an equivalence of homotopy theories. 
Remarks. 1. See Appendix A for a proof. This statement only becomes significant if we define fibrations and cofibrations in the two categories:

(a) fibrations and cofibrations in $0-$ Split $-\pi$ are defined exactly as equivariant fibrations and cofibrations in the category of simplicial sets.

(b) a cofibration in (DGC- $\pi)_{2}$ is defined to be an inclusion of DGA-coalgebras that is an inclusion of a direct summand;

(c) a fibration in (DGC- $\pi)_{2}$ is essentially a projection of a twisted tensor product (up to a weak equivalence).

2. This implies that the equivalence of categories essentially maps fibrations of spaces into twisted tensor products of DGA-coalgebras (up to a homotopy equivalence). This fact is the essential ingredient used in the construction of minimal models for objects in 0-Split- $\pi$.

Definition 1.9. Let $\overparen{K(\pi, 1)}$ denote the universal cover of $K(\pi, 1)$ (regarded as a simplicial set)-this is equipped with a free $\pi$-action. Let $\mathfrak{B}(\pi)$ denote the result of collapsing the 1-skeleton of $\widetilde{K(\pi, 1)}$ and let $\mathfrak{p}(\pi): \widetilde{K(\pi, 1)} \rightarrow \mathfrak{B}(\pi)$ denote the collapsing map. Then $\mathfrak{B}(\pi)$ is an object in PointedFree- $\pi$.

Remarks. Note that the homotopy type of $\mathfrak{B}(\pi)$ in PointedFree- $\pi$ is uniquely defined and that $\mathfrak{B}(\pi)$ is functorial in $\pi$ (at least if we always use the Eilenberg-Mac Lane description of $K(\pi, 1)$ given in [6]).

Definition 1.10. Let $\mathrm{Map}_{2}-\pi$ denote the category of maps $f: X \rightarrow$ $K(\pi, 1)$ with the following properties:

1. $X$ is a simplicial set;

2. the map $f$ induces an isomorphism of 1-skeleta and of fundamental groups. Morphisms are commutative triangles:

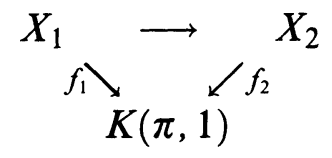

Remarks. Given any simplicial set $Z$ with $\pi_{1}(Z)=\pi$, it is well known that $Z$ is weakly equivalent to an object of $\mathrm{Map}_{2}-\pi$. For instance construct the semi-simplicial minimal model for $Z$ as in [5].

Definition 1.11. Let PointedMap- $\pi$ denote the category of maps $f: X \rightarrow \mathfrak{B}(\pi)$, where $X \in$ PointedFree- $\pi$ and a morphism $\left(f_{1}: X_{1} \rightarrow \mathfrak{B}(\pi)\right) \rightarrow\left(f_{2}: X_{2} \rightarrow \mathfrak{B}(\pi)\right)$ is defined to be a commutative 
triangle

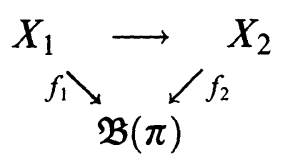

Definition 1.12. We define functors $\mathfrak{H}: \mathrm{Map}_{2}-\pi \rightarrow$ PointedMap- $\pi$ and $\mathfrak{J}:$ PointedMap- $\pi \rightarrow \mathrm{Map}_{2}-\pi$ as follows:

(a) Given an object $c: X \rightarrow K(\pi, 1)$ of $\mathrm{Map}_{2}-\pi$ let $\tilde{c}: \tilde{X} \rightarrow \widetilde{K(\pi, 1)}$ be the induced map of universal covering spaces. Now collapse the 1-skeleta of $\tilde{X}$ and $\widetilde{K(\pi, 1)}$ to get a map to PointedFree- $\pi$ $\tilde{c}^{\prime}: \tilde{X} / 1$-skeleton $\rightarrow \mathfrak{B}(\pi)$. This map is $\mathfrak{H}(X)$. This construction is clearly functorial and preserves weak equivalences.

(b) Given an object $a=(f: X \rightarrow \mathfrak{B}(\pi))$ of PointedMap- $\pi$ define $\mathfrak{J}(a)$ to be $P(X) / \pi$, where $P(X)$ is the equivariant fibered product over the diagram

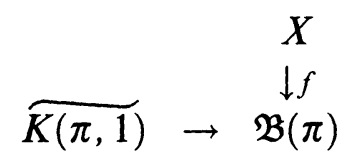

where the bottom row is the canonical map $\overparen{K(\pi, 1)} \rightarrow \mathfrak{B}(\pi)$. The total space of the fibered product is equipped with a free $\pi$-action.

Proposition 1.13. The functor $\mathfrak{H}$ induces an equivalence of categories $\mathfrak{H}: \mathrm{Map}_{2}-\pi \rightarrow$ PointedMap- $\pi$.

Proof. Consider $\mathfrak{J} \circ \mathfrak{H}(X)$, where $X \in \operatorname{Map}_{2}-\pi$. The universal property of the cartesian square that results from forming a fibered product implies the existence of a natural map $X \rightarrow \mathfrak{J} \cdot \mathfrak{H}(X)$.

Claim. This map is an isomorphism of simplicial sets.

Proof of claim. This follows from:

(a) Since $\widetilde{K(\pi, 1)} \rightarrow \mathfrak{B}(\pi)$ is an isomorphism above dimension 1 , $X \rightarrow \mathfrak{J} \circ \mathfrak{H}(X)$ will be an isomorphism above dimension 1 ;

(b) Since $\tilde{c}: \tilde{X} \rightarrow \widetilde{K(\pi, 1)}$ is an isomorphism below dimension 2 (by statement 2 in 1.10), $X \rightarrow \mathfrak{J} \circ \mathfrak{H}(X)$ will be an isomorphism below dimension 2.

A similar argument implies that $\mathfrak{H} \circ \mathfrak{J}$ is an isomorphism. The conclusion follows. 
COROllary 1.14. Given an object $X$, of $\mathrm{Map}_{2}-\pi$ it is possible to associate a $\mathbb{Q}$-model that is a homomorphism of DGA-coalgebras $M(\mathfrak{H}(X)) \rightarrow M(\mathfrak{B}(\pi))$, where $M(\mathfrak{B}(\pi))$ depends only upon the group $\pi$. Two such objects are homotopy equivalent if and only if there exists a commutative diagram

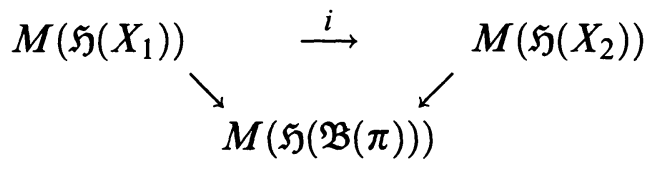

where the map $i$ is an isomorphism of DGA-coalgebras.

We can also apply this result to the question of realizability of $\mathbb{Q} \pi$ chain-complexes.

DeFINITION 1.15. Let $C$ be a projective $\mathbb{Q} \pi$-chain complex such that $H_{0}(C)=\mathbb{Q}, H_{1}(C)=0$ and let $c: C \rightarrow \widetilde{K(\pi, 1)}$ be the canonical map that induces an isomorphism of homology in dimension 0 . Define $C_{\mathfrak{B}}$ as follows:

(a) Form the algebraic mapping cone $\mathfrak{A}(c)$;

(b) form the map $i: \widetilde{K(\pi, 1)} \rightarrow \mathfrak{A}(c)$ sending $\overparen{K(\pi, 1)}$ to the imbedded copy of itself via the identity map;

(c) homotope $i$ to a map, $i^{\prime}$, that sends the 1-skeleton of $\widetilde{K(\pi, 1)}$ to 0 -this is possible because $c$ is a chain-homotopy equivalence below dimension 2 so that $\mathfrak{A}(c)$ is contractible below dimension 2;

(d) $C_{\mathfrak{B}}$ is defined to be $\Sigma^{-1} \mathfrak{A}\left(i^{\prime}\right) /(1$-skeleton of $\widetilde{K(\pi, 1)}) \oplus \mathbb{Q}$.

REMARKs. By abuse of notation, we have identified $\widetilde{K(\pi, 1)}$ with its chain-complex. The copy of $\mathbb{Q}$ that is added is $\left(C_{\mathfrak{B}}\right)_{0}$.

2. Essentially, $\sum^{-1} \mathfrak{A}\left(i^{\prime}\right)$ turns out to be chain-homotopy equivalent to $C$ but it has an imbedded copy of the 1-skeleton of $\widetilde{K(\pi, 1)}$ (this is guaranteed by the fact that we modified $i$ by a homotopy giving a map that sends the 1 -skeleton of $\widetilde{K(\pi, 1)}$ to 0$)$.

3. Note that, although it appears that arbitrary choices were made in the selection of $i^{\prime}, C_{\mathfrak{B}}$ is uniquely defined up to an isomorphism of chain-complexes. This is due to the well-known fact that varying a map by a chain-homotopy alters the algebraic mapping cone by an isomorphism. In fact two different choices give rise to equivalent objects in the category of maps of chain-complexes to the chain-complex of $\mathfrak{B}(\pi)$.

Proposition 1.16. If the chain-complex of a simplicial set $X$ is $C$, then the map of complexes induced by $\mathfrak{H}(X)$ is equivalent to $C_{\mathfrak{B}} \rightarrow \mathfrak{B}(\pi)$ in the category of maps of chain-complexes to that of $\mathfrak{B}(\pi)$. 
Proof. We start with the following diagram (which commutes up to a homotopy):

$$
C(\tilde{X}) \underset{c_{1} \searrow \underset{K(\pi, 1)}{\swarrow c_{2}}}{\longrightarrow} C
$$

where $C(\tilde{X})$ is the chain-complex of the universal covering space of $X$, the horizontal map is a chain-homotopy equivalence and the maps $c_{i}$ induce homology isomorphisms in dimension 0 . We get the following diagram of algebraic mapping cones:

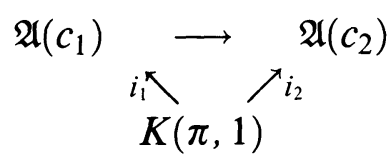

where the $i$-maps are the inclusions, and the horizontal map is, again, a chain-homotopy equivalence. This gives rise to the following chainhomotopy-commutative diagram:

$$
\begin{array}{ccc}
\Sigma^{-1} \mathfrak{A}\left(i_{1}^{\prime}\right) & \stackrel{v}{\longrightarrow} & \Sigma^{-1} \mathfrak{A}\left(i_{2}^{\prime}\right) \\
h \downarrow & \searrow p & \overparen{K(\pi, 1)} \\
C(\tilde{X}) & &
\end{array}
$$

Here $v$ is a chain-homotopy equivalence, $p$ is the standard projection, and $h$ is the projection to $C(\tilde{X})$, modified to send the 1-skeleton of $\widetilde{K(\pi, 1)}$ to that of $C(\tilde{X})$. After factoring out the 1-skeleton of all chain-complexes, we end up with chain-homotopy equivalences in the vertical and horizontal maps.

Proposition 1.17. Let $f_{i}: C_{i} \rightarrow \overparen{K(\pi, 1)}, i=1,2$, be two chainmaps of $\mathbb{Q} \pi$-projective chain-complexes and suppose the $f_{i}: 1$. induce isomorphisms in dimensions 0 and 1; and, 2. induce isomorphisms in homology in dimension 1. Suppose, in addition, we get the following commutative diagram (up to a chain-homotopy) :

1.18:

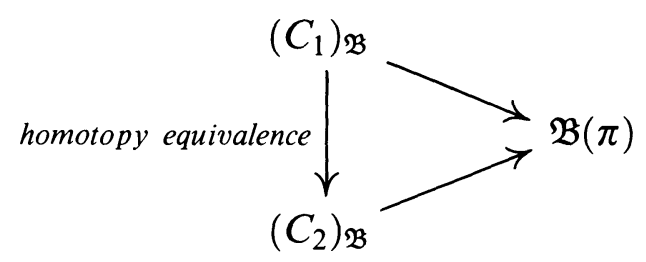


Then there exists a map $g: C_{1} \rightarrow C_{2}$ that makes the following diagram commute up to a chain-homotopy:

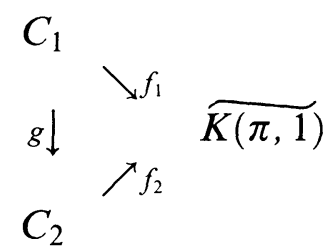

REMARKs. Consider the isomorphism $j: \widetilde{K(\pi, 1)_{2}} \rightarrow \mathfrak{B}(\pi)_{2}$. Given the induced map of chain-complexes, $f: C \rightarrow \mathfrak{B}(\pi)$, of $H(X)$ we can reconstruct the chain-complex of $X$ as follows:

$$
\begin{gathered}
C(X)_{i}=C_{i}, \quad \text { for } i>1 ; \quad C(X)_{i}=\widetilde{K(\pi, 1)}, \quad \text { for } i \leq 1 . \\
\partial=\partial_{\widetilde{K(\pi, 1)}} \cdot j^{-1}: C(X)_{2} \rightarrow C(X)_{1} .
\end{gathered}
$$

The conclusion follows.

THEOREM 1.19. Let $C$ be a $\mathbb{Q} \pi$-chain complex such that $H_{0}(C)=\mathbb{Q}$, $H_{1}(C)=0$. Let $c_{\mathfrak{B}}: C_{\mathfrak{B}} \rightarrow \mathfrak{B}(\pi)$ the associated chain-complex and map. Then $C$ is topologically realizable if and only if there exists a $\mathbb{Q}$-model $M$ and a DGA-coalgebra homomorphism $f: M \rightarrow M(\mathfrak{B}(\pi))$ such that the following diagram commutes up to a chain-homotopy:

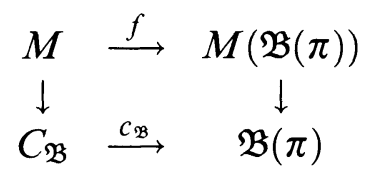

REMARKS. The vertical maps are $\mathbb{Q} \pi$-chain homotopy equivalences.

Proof. Necessity is clear. Sufficiency follows from the fact that:

(a) $M$ is the $\mathbb{Q}$-model of some topological space $Y$ with a map $Y \rightarrow$ $\mathfrak{B}(\pi)$;

(b) we form $X=\mathfrak{J}(Y \rightarrow \mathfrak{B}(\pi))$. This is a space with the property that $C(\mathfrak{H}(X)) \approx C(\tilde{X})_{\mathfrak{B}} \rightarrow \mathfrak{B}(\pi) \approx C_{\mathfrak{B}} \rightarrow \mathfrak{B}(\pi)$ (by 1.16) (where $C(*)$ stands for chain-complex and $\approx$ stands for "chain-homotopyequivalent to"). Proposition 1.17 then implies that $C(\tilde{X})$ is chainhomotopy equivalent to $C$.

Let $I_{2}(\pi)=H_{2}(\mathfrak{B}(\pi))=\operatorname{kernel} \mathfrak{B}(\pi)_{2} \stackrel{\partial}{\rightarrow} \mathfrak{B}(\pi)_{1}$. The map $C_{\mathfrak{B}} \rightarrow$ $\mathfrak{B}(\pi)$ induces a map $H_{2}\left(C_{\mathfrak{B}}\right) \rightarrow I_{2}(\pi)$ and implies the existence of a module extension $H_{2}(C) \rightarrow H_{2}\left(C_{\mathfrak{B}}\right) \rightarrow I_{2}(\pi)$, classified by an element of $\operatorname{Ext}_{\mathbb{Q} \pi}^{1}\left(I_{2}(\pi), H_{2}(C)\right)=H^{3}\left(\pi, H_{2}(C)\right)$. This extension characterizes the contribution of the first homological $k$-invariant of $C$ and it turns 
out that:

Proposition 1.20. The $\mathbb{Q} \pi$-chain complex $C$ is topologically realizable if and only if there exists a map of $\mathbb{Q}$-models $f: Y \rightarrow M(\mathfrak{B}(\pi))$ such that: 1 . the underlying chain-complex of $Y$ is chain-homotopy equivalent to $C$, and; 2. the map $H_{2}(Y) \rightarrow I_{2}(\pi)$ induced by $f$ defines the same extension as that of $C$.

Proof. Clearly the topological realizability of $C$ implies the hypotheses. On the other hand, if the hypotheses are satisfied we can also satisfy the hypotheses of 1.19 since $\mathfrak{B}(\pi)$ is essentially a suspension of a resolution and chain-maps to resolutions are characterized (up to a chain-homotopy) by the induced maps in homology.

2. Q-models-the split case. This section will develop $\mathbb{Q}$-models algebraically and show that the homotopy theory of DGA-coalgebras produced by Quillen's theory can be represented by $\mathbb{Q}$-models. We will use the results of Appendix B extensively.

DEFINITION 2.1. Let $M$ be an abelian group, and let $n$ be an integer $>$ 1. Then:

(a) if $n$ is even $U(M, n)$ is defined to be the divided power algebra defined in [5] as follows:

(i) $U(M, n)_{k}$ vanishes if $k$ is not a multiple of $n$;

(ii) $U(M, n)_{n t}$ is generated by symbols $\gamma_{t}(m)$ for all $m \in M$ and these symbols satisfy the relations: $\gamma_{0}(m)=1 \in U(M, n)_{0}=\mathbf{Z}$; $\gamma_{\alpha}(m) \cdot \gamma_{\beta}(m)=(\alpha+\beta) ! / \alpha ! \beta ! \gamma_{\alpha+\beta}(m)$, for all $m \in M$ and $\alpha, \beta \geq 0$; $\gamma_{t}\left(m_{1}+m_{2}\right)=\sum_{\alpha+\beta=t} \gamma_{\alpha}\left(m_{1}\right) \cdot \gamma_{\beta}\left(m_{2}\right) ; \gamma_{t}(r m)=r^{t} \gamma_{t}(r m)$, for all $m \in M$ and $r \in \mathbb{Z}$.

(b) if $n$ is odd $U(M, n)$ is defined to be the exterior algebra on $M$ concentrated in dimensions that are multiples of $n$.

Remarks. 1. Note that if $n$ is even $U(M, n)_{2 n}=\Gamma(M)$, the Whitehead "universal quadratic" functor.

2. Note that the relations above are natural in the sense that, if $M$ is a $\mathbb{Q} \pi$-module, then $U(M, n)$ is equipped with a natural $\mathbb{Q} \pi$-model structure, and the multiplication respects this structure.

3. $U(M, n)$ is also equipped with a commutative coproduct operation -i.e. a map $\Delta: U(M, n) \rightarrow U(M, n) \otimes U(M, n)$ defined by $\Delta\left(\gamma_{t}(m)\right)=$ $\sum_{\alpha+\beta=t} \gamma_{\alpha}(m) \otimes \gamma_{\beta}(m)$, if $n$ is even and

$$
\Delta\left(m_{1} \wedge \cdots \wedge m_{t}\right)=\left(1 \otimes m_{1}+m_{1} \otimes 1\right) \wedge \cdots \wedge\left(1 \otimes m_{t}+m_{t} \otimes 1\right),
$$


where $(a \otimes b) \wedge(c \otimes d)$ is defined to be equal to

$$
(-1)^{\operatorname{dim}(b) \cdot \operatorname{dim}(c)}(a \wedge c) \otimes(b \wedge d),
$$

if $n$ is odd. With this coproduct it becomes a DGA-Hopf algebra.

Proposition 2.2. Let $(C, \Delta)$ be a DGA-coalgebra whose lowestdimensional (above dimension 0) nonvanishing homology module is $M$ in dimension $n>1$. Then there exists a homomorphism of DGAcoalgebras $f: C \rightarrow \mathfrak{U}(M, n, Q)$ inducing an isomorphism of homology in dimension $n$.

Proof. Let $g: C \rightarrow \Sigma^{n} M_{*}$ be a chain-map inducing an isomorphism of homology in dimension $n-g$ exists because its target is a resolution. Let $\Delta^{\prime}=\Delta-I$, where $I$ is the trivial coproduct sending $x \in C$ to $1 \otimes x+x \otimes 1$. If $\eta$ is the augmentation of $C$ then $^{2} f=$ $\eta+g+(g \otimes g) \circ \Delta^{\prime}+(g \otimes g \otimes g) \circ\left(1 \otimes \Delta^{\prime}\right) \cdot \Delta^{\prime}+\cdots$ is a DGA-coalgebra homomorphism from $C$ to the symmetric algebra $S\left(\Sigma^{n}\left(M_{*}\right)=\mathfrak{U}(M, n, Q)\right.$ with the required properties (see B.8 in Appendix B).

Proposition 2.3. Let $C$ be a commutative DGA-coalgebra $A$ a commutative DGA-algebra and let $\alpha: C \rightarrow \Sigma A$ be a chain-map. Let $t\left(\alpha^{\prime}\right): C$ $\rightarrow A$ be defined by $t\left(\alpha^{\prime}\right)(x)=(-1)^{\operatorname{dim}(x)-1} \alpha(x)$. Then $t\left(\alpha^{\prime}\right): C \rightarrow A$ defines a twisting cochain.

REMARK. See B.7 in Appendix B for a proof.

Definition 2.4. Let $M$ be a $\mathbb{Q} \pi$-module and let $M_{*}$ be the standard resolution of $M$ defined in B.1. Then $\mathfrak{U}(M, n, Q)$ is defined to be $\stackrel{\lim }{\longrightarrow} V_{i}$, where $V_{i}$ is a direct system constructed as follows:

1. $V_{j, j}=\mathfrak{U}\left(M_{j}, n+j\right)$

2. $V_{j, k}=V_{j, k+1} \otimes_{\alpha_{k+1}} U\left(M_{k}, n+k\right)$, where $j \geq k+1$ and $\alpha_{i+1}$ is the twisting cochain defined by the map $\partial_{i+1} \otimes 1: Q_{i+1} \otimes M \rightarrow Q_{i} \otimes M$.

3. $V_{i}=V_{i, 0}$.

REMARKS. 1. $\mathfrak{U}(M, n, Q)$ is just an iterated twisted tensor product of $U\left(M_{i}, n+i\right)$ 's that reflect the structure of the standard resolution of $M$. The only reason that we use a direct limit is that the standard resolution of $M$ may be infinite dimensional.

\footnotetext{
${ }^{2}$ Compare this with Brown's definition of the classifying map of a twisted tensor product at the end of [3].
} 
2. $\mathfrak{U}(M, n, Q)$ supports the untwisted product and coproduct structures, by B.11 and B.10 in Appendix B. Note that $\mathfrak{U}(M, n, Q)$ is canonically isomorphic to the symmetric (Hopf) algebra $S\left(\Sigma^{n} M_{*}\right)$, by B.9 in Appendix B.

3. $\mathfrak{U}(M, n, Q)$ is essentially a model for a $K(M, n)$ in the homotopy category $(\mathrm{DGC}-\pi)_{2}$. It can also be regarded as a dual to a simple polynomial algebra (in analogy with Sullivan's "Infinitesimal Computations in Topology"), in which case the twisted tensor products are duals of Hirsch Extensions.

Definition 2.5. A $\mathbb{Q}$-model, $T$, is defined to be a sequence $\left\{T_{n}\right\}$ of DGA-coalgebras constructed as follows:

1. $T_{0}=T_{1}=\mathbb{Q}$;

2. for all $i>1 T_{i+1}=T_{i} \otimes_{t(f)} \mathfrak{U}\left(M_{i+1}, i+1, Q\right)$, where $f$ is some chain-map $f: T_{i} \rightarrow \Sigma^{i+1}\left(M_{i+1}\right)_{*}$. See the discussion preceding B.13 in Appendix B. B.15 implies that $T_{i+1}$ is really determined (as a DGAcoalgebra) by the class of $t(f) \in H^{i+1}\left(T_{i}, M_{i+1}\right)$.

The sequence of modules $\left\{M_{i}\right\}$ will be called the homotopy modules of the $\mathbb{Q}$-model and the twisting cocycles used to form the twisted tensor products above, will be called its $k$-invariants.

3. The inverse limit of the $T_{i}$ is called the total space of the $\mathbb{Q}$ model $T$.

Remarks. See 2.3 for a definition of $t(*)$. Note that each of the twisted tensor products will support the untwisted coproduct structuresee B.10 in Appendix B. We use this coproduct structure in building the higher stages of the $\mathbb{Q}$-model (since they are twisted tensor products-which are defined with respect to some coproduct).

Definition 2.6. Let $C$ be a commutative DGA-coalgebra. Then the map $m: C \rightarrow C \otimes C$ that sends $a \in C$ to $1 \otimes a+a \otimes 1 \in C \otimes C$ is a chain-map. Define $P(C)$, the primitive subcomplex of $C$, to be the kernel of the chain-map $\Delta-m: C \rightarrow C \otimes C$.

REMARKs. Let $\alpha: \mathfrak{U}(M, n, Q) \rightarrow \Sigma \mathfrak{U}(M, n-1, Q)$ be the chain-map that is the identity map on $P(\mathfrak{U}(M, n, Q))=\Sigma^{n} M_{*}$ (except that it lowers dimension by 1) and vanishes on all elements of degree $>n$. Then $t(\alpha): \mathfrak{U}(M, n, Q) \rightarrow \mathfrak{U}(M, n-1, Q)$ is a twisting cochain with the property that $\mathfrak{U}(M, n, Q) \otimes_{t(\alpha)} \mathfrak{U}(M, n-1, Q)$ is acyclic. This follows from B.9 in Appendix B, which implies that

$$
\mathfrak{U}(M, n, Q) \otimes_{t(\alpha)} \mathfrak{U}(M, n-1, Q) \cong S\left(\Sigma^{n} M_{*} \oplus_{\alpha^{\prime}} \Sigma^{n-1} M_{*}\right),
$$

where $\alpha^{\prime}: \Sigma^{n} M_{*} \rightarrow \Sigma^{n-1} M_{*}$. 
Proposition 2.7. Let $(C, \Delta)$ be a DGA-coalgebra. Then there exists $a$ Q-model $T$ such that $(C, \Delta)=T$, as objects of $\mathrm{Ho}\left((\mathrm{DGC}-\pi)_{2}\right)$.

REMARKs. Recall the definition of equality of objects in a localized category in Remark 2 following 1.4.

Proof. There are several ways to prove this result. We can use the equivalence of homotopy theories in 1.8 and the existence and uniqueness of Postnikov Towers in usual homotopy theory, for instance. In this case the fibrations in the Postnikov Tower map into twisted tensor products of the $\mathfrak{U}(M, n, Q)$. We will give a purely algebraic proof using 2.2. We use induction on the stages of the construction of a $\mathbb{Q}$-model for $C$. We will show that there exists a sequence of DGA-coalgebras $F_{i}, i>0$, with $F_{0}=C$, maps $g_{i}: F_{i} \rightarrow F_{i-1}$ such that:

1. every $g_{i}$ is a DGA-coalgebra homomorphism and a homology equivalence;

2. there exists an $i$-connected homomorphism of DGA-coalgebras $h_{i}: F_{i} \rightarrow T_{i}$, where $T_{i}$ is the $i$ th stage of a $\mathbb{Q}$-model.

Assume that we are given $h_{i-1}: F_{i-1} \rightarrow T_{i-1}$. We will construct the next stage of $T$ and $F_{i}$. Let $\Omega T_{i-1}$ denote "Quillen's cobar construction of $T_{i-1}$ "-this is defined on p. 290 of [11]. Recall that this is the universal enveloping algebra of a DG-Lie algebra and it has the property that $T_{i-1} \otimes_{S} \Omega T_{i-1}$ (untwisted coproduct structure) is acyclic in positive dimensions, where $s: T_{i-1} \rightarrow \Omega T_{i-1}$ is Quillen's Universal Twisting function. We form the pullback $P_{i-1}=F_{i-1} \otimes_{h_{t-1} \text { os }}$ $\Omega T_{i-1}$. Now $P_{i-1}$ is at least $i$-connected. Consider the twisting cochain $y: T_{i-1} \rightarrow P_{i-1}$-it is essentially identical to $s: T_{i-1} \rightarrow \Omega T_{i-1}$, i.e. its image lies entirely in $1 \otimes \Omega T_{i-1}$. Let $\eta_{1}$ and $\eta_{2}$ denote the augmentations of $T_{i-1}$ and $\Omega T_{i-1}$, respectively. It is not hard to see that $\eta_{1} \otimes 1 \otimes \eta_{2}: T_{i-1} \otimes_{y} P_{i-1} \rightarrow F_{i-1}$ is a homology equivalence-this map is $g_{i}$ and $T_{i-1} \otimes_{y} P_{i-1}$ is $F_{i}$. Let $z_{i}: P_{i-1} \rightarrow \mathfrak{U}\left(M_{i}, i, Q\right)$ be a DGAcoalgebra homomorphism inducing an isomorphism in homology in dimension $i$-it exists by 2.2. The composite $z_{i} \circ y: T_{i-1} \rightarrow \mathfrak{U}\left(M_{i}, i, Q\right)$ will also be a twisting cochain (by B.7 in Appendix B) and there will exist a DGA-coalgebra homomorphism $h_{i}: F_{i-1} \otimes_{y} \mathfrak{U}\left(M_{i}, i, Q\right)=F_{i} \rightarrow$ $F_{i-1} \otimes_{z_{1} \circ y} \mathfrak{U}\left(M_{i}, i, Q\right)$, which will also be $i$-connected. This completes the proof of the inductive step and the result.

Proposition 2.8. Let $T$ be a $\mathbb{Q}$-model. The homology modules of $P(T)$ are the homotopy-modules of $T$. 
Proof. This follows from the fact that $P(\mathfrak{U}(M, n, Q))=\Sigma^{n} M_{*}$ and the fact that $T$ has the untwisted coproduct structure.

Proposition 2.9. Let $f: T_{1} \rightarrow T_{2}$ be a DGA-coalgebra isomorphism of the total spaces of two $\mathbb{Q}$-models. Then $f$ is of the form $S\left(f \mid P\left(T_{1}\right)\right)$.

Proof. Let $T$ be a $\mathbb{Q}$-model. Then $T$ can be written as a tensor product of the form

$$
\bigotimes_{i=1}^{\infty} \bigotimes U\left(M_{i} \otimes P_{i, j}, i\right)=\bigotimes_{i=1}^{\infty} U\left(\bigoplus M_{i} \otimes P_{i, j}, i\right)
$$

(this doesn't respect the differential).

Claim. The map $f$ respects this tensor product decomposition.

The conclusion follows from the fact that elements of the $i$ th factor of $T$ can be characterized as follows: An $n$-dimensional element, $e$, is in $T_{i} f$ if and only if all factors in the terms of $\Delta^{n}(e)$ are of dimension $k i$ (for some value of $k$ ) or dimension 0 . This property must be preserved under any isomorphism of DGA-coalgebras.

REMARKS. 1. We are not claiming that any map of primitive subcomplexes of $\mathbb{Q}$-models induces a map of the $\mathbb{Q}$-models.

2. Note that this implies that if we equip $\mathbb{Q}$-models with the tensor product algebra structure (this doesn't necessarily make them DGAalgebras) this algebra structure will be preserved by any DGA-coalgebra isomorphism.

3. If the map $f: T_{1} \rightarrow T_{2}$ is only a DGA-coalgebra homomorphism then an element $x$ of $T_{1}$ of dimension $i$ can map to elements of $T_{2}$ of dimension $k i$. Consider a map $\mathfrak{U}(M, 2, Q) \rightarrow \mathfrak{U}(\Gamma(M), 4, Q)$ inducing an isomorphism of homology in dimension $4 .^{3}$

THEOREM 2.10. Let $f: T_{1} \rightarrow T_{2}$ be an DGA-coalgebra homomorphism of the total spaces of two $\mathbb{Q}$-models. Then there exist isomorphisms of $\mathbb{Q}$-models $\mathscr{I}_{i}: T_{i} \rightarrow T_{i}, i=1,2$, and a DGA-coalgebra homomorphism $\hat{f}: T_{1} \rightarrow T_{2}$ such that the following diagram commutes:

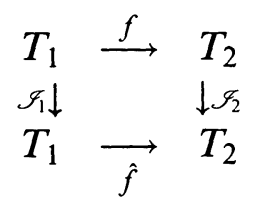

${ }^{3}$ The existence of such a map is guaranteed by B.19. 
The map $\hat{f}$ has the following properties:

1. if $p_{i, j}: T_{i} \rightarrow\left(T_{i}\right)_{j}$ is the projection to the $j$ th stage the following diagram commutes:

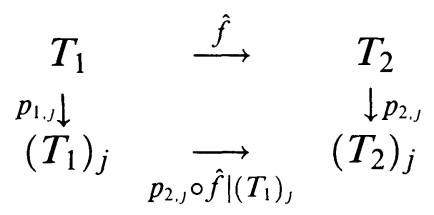

2. $p_{2, j+1} \circ \hat{f}\left|\left(T_{1}\right)_{j+1}=p_{2, j} \circ \hat{f}\right|\left(T_{1}\right)_{j} \otimes S\left(f_{j+1}^{*} \otimes 1\right)+\Xi_{j+1} \cap^{*}:\left(T_{1}\right)_{j+1}=$ $\left(T_{1}\right)_{j} \otimes \mathfrak{U}\left(M_{j+1}, j+1, Q\right) \rightarrow\left(T_{2}\right)_{j+1}=\left(T_{2}\right)_{j} \otimes \mathfrak{U}\left(M_{j+1}^{\prime}, j+1, Q\right)$.

Remarks. 1. The map $S\left(f_{j+1}^{*} \otimes 1\right)$ is the map induced by $f \mid P\left(T_{1}\right)$.

2. The map $\hat{f}$ will be called the uniformization of $f$.

3. See Appendix $\mathrm{C}$ for a proof.

COROllary 2.11. Let $f: T_{1} \rightarrow T_{2}$ be a DGA-coalgebra homomorphism of the total spaces of two $\mathbb{Q}$-models that induces isomorphisms in homology. Then the uniformization, $\hat{f}$, of $f$ is an isomorphism of DGA-coalgebras.

Remarks. This follows from the fact that the maps $S\left(f_{j+1}^{*} \otimes 1\right)$ will all be isomorphisms and the term $\Xi_{j+1} \cap^{*}$ will look like a lower triangular matrix, and so, will be an isomorphism.

COROllary 2.12. Two Q-models are equal as objects of $\mathrm{Ho}\left((\mathrm{DGC}-\pi)_{2}\right)$ if and only if they are isomorphic as DGA-coalgebras.

A topological realization of a $\mathbb{Q}-$ model, $T$, is defined to be a topological space $X$ with a chain-homotopy equivalence $h_{i}: C\left(X_{i}\right) \rightarrow T_{i}$ for all $i$, where $X_{i}$ is the total space of the $i$ th stage of a Postnikov tower for $X$. In addition, we assume that for all $i p_{i} \circ h_{i}=h_{i-1} \circ q_{i-1}$, where $p_{i}: T_{i} \rightarrow T_{i-1}, q_{i}: X_{i} \rightarrow X_{i-1}$ are the projections and $H_{i}\left(\mathfrak{A}\left(q_{i}\right)\right) \cong$ $H_{i}\left(\mathfrak{A}\left(p_{i}\right)\right)$.

Proposition 2.13. Let $T$ be a $\mathbb{Q}$-model. Then $T$ has a topological realization.

Proof. This follows from the basic result 1.4. Simply construct a simplicial set, $X$, realizing the underlying DGA-coalgebra of the total space of $T$. This construction can be carried out in a step-by-step fashion, building a Postnikov Tower for $X$ using the equivalence of 
homotopy theories in 1.8 and B.18. The equivalence of homotopy theories in 1.8 implies that the fibrations in the Postnikov tower translate into twisted tensor products in $\mathrm{Ho}\left((\mathrm{DGC}-\pi)_{2}\right)$. Theorem B.18 then implies that the "Eilenberg-MacLane" spaces, correspond to the $\mathfrak{U}(M, n, Q)$.

Definition 2.14. Let $f: X_{1} \rightarrow X_{2}$ be a map between two 0 -split $\mathrm{CW}$-complexes. Then $f$ is a rational homotopy equivalence if:

1. $f$ induces an isomorphism of fundamental groups;

2. $f$ induces isomorphisms $\pi_{i}(f) \otimes \mathbb{Q}: \pi_{i}\left(X_{1}\right) \otimes \mathbb{Q} \rightarrow \pi_{i}\left(X_{2}\right) \otimes \mathbb{Q}$, for $i \geq 2$.

Proposition 2.15. Let $T\left(X_{1}\right), T\left(X_{2}\right)$ be $\mathbb{Q}$-models of two 0-split $C W$-complexes. Then $X_{1}$ and $X_{2}$ are rationally homotopy equivalent if and only if $T\left(X_{1}\right), T\left(X_{2}\right)$ are isomorphic as DGA-coalgebras.

Proof. Clearly, if the spaces are rationally homotopy equivalent the $\mathbb{Q}$-models will be isomorphic-by 2.10. Conversely, if the $\mathbb{Q}$-models are isomorphic we can build Postnikov towers for the two spaces that are rationally homotopy equivalent.

We will now show how some well-known topological invariants of a space can be computed from its $\mathbb{Q}$-model. Note that a $\mathbb{Q}$-model comes equipped with a product structure as well as a coproduct structurethis results from the twisted tensor product decomposition of the $\mathbb{Q}$ model. We just equip the twisted tensor product with the tensor product multiplication (which exists since each factor is a DGA-Hopf algebra). The $\mathbb{Q}$-model will generally not be a DGA-algebra with respect to this product structure.

Proposition 2.16. Let $T$ be $a$ Q-model and let

$$
P^{2}(T)=\Delta^{-1}(P(T) \otimes P(T))
$$

Then the product operation described above defines a map $\mu: P(T) \otimes$ $P(T) \rightarrow P^{2}(T)$. This map is natural with respect to DGA-coalgebra homomorphisms of $T$.

Proof. This follows from the fact that we can express $\mu$ as $\Delta^{-1} \circ \mathscr{S}$, where $y: C \otimes C \rightarrow C \otimes C$ is the symmetrization map that sends $x \otimes y$ to $\left(x \otimes y+(-1)^{\operatorname{dim}(x) \operatorname{dim}(y)} y \otimes x\right) / 2$.

Note that the natural product operation won't preserve the differential of the $\mathbb{Q}$-model. The degree to which it fails to do so turns out to be significant. 
Proposition 2.17. Let $T$ be a $\mathbb{Q}$-model. If $x, y \in P(T)$ let $[x, y]=$ $(d \mu)(x \otimes y)=d \circ \mu(x \otimes y)-\mu(d(x \otimes y))$. Then [, ] defines the structure of $a$ DG-Lie algebra on $\Sigma^{-1} P(T)$.

Remarks. 1. We have to desuspend $P(T)$ so that the dimensions of products will be right.

2. Since the homology modules of $P(T)$ coincide with the homotopy modules of $T$, this Lie algebra operation defines a product on homotopy modules.

Proof. We first have to verify that $[x, y] \in P(T)$. To see this note that the product operation $\mu$ preserves the untwisted tensor product differential. It follows that in forming $d \mu$, the only terms that are significant are those involving twisting cochains. But the images of twisting cochains used to form $T$ from $\mathfrak{U}(M, n, Q)$ 's all lie in the primitive subcomplexes of higher terms. The other properties of a DGALie algebra (for instance the Jacobi identity) follow easily from the definition.

Proposition 2.18. The product operation induced on homotopy modules by the Lie bracket operation on $\Sigma^{-1} P(T)$ defined above, coincides with the Whitehead product.

Proof. Recall the definition of the Whitehead product: Given homotopy classes $x \in \pi_{i}(X): S^{i} \rightarrow X, y \in \pi_{j}(X): S^{j} \rightarrow X$, represented by maps $f_{x}: S^{i} \rightarrow X$ and $f_{y}: S^{j} \rightarrow X$ the Whitehead product is defined to be the image of a suitable generator of $\pi_{i+j-1}\left(S^{i} \vee S^{j}\right)$ under the map $f_{x} \vee f_{y}: S^{i} \vee S^{j} \rightarrow X$. The generator in question is the one that can be used to attach a cell to $S^{i} \vee S^{j}$ to form $S^{i} \times S^{j}$. The conclusion now follows from the naturality of the Lie bracket operation we have defined and direct computation, using a $\mathbb{Q}$-model for $S^{i} \times S^{j}$.

These results give some insight into the meaning of Whitehead products. The structure constants of the Lie algebra structure we have defined are determined by some of the twisting cochains used to form a Q-model (see the proof of 2.17). Roughly speaking, Whitehead products measure the quadratic structure of the $\mathbb{Q}$-model and nothing more. Quillen's paper [11] defines a DG-Lie algebra that is similar to ours ${ }^{4}$, and proves that Whitehead products are determined by this Lie algebra structure and it is tempting to compare his construction with ours. It

\footnotetext{
${ }^{4}$ This is the functor $\mathscr{L}(C)$-Quillen's Lie-algebra version of the cobar-construction.
} 
turns out that the two constructions are not equivalent-and Quillen's contains more information than ours. The underlying chain-complex of the DG-Lie algebra $\Sigma^{-1} P(T)$ turns out to be chain-homotopy equivalent to the underlying chain-complex of the DG-Lie algebra defined by Quillen but the Lie algebra structure is not quite the same (although they both induce the same Lie algebra structure on homology). The Lie algebra defined by Quillen determines the original DGA-coalgebra (up to a homotopy equivalence), whereas $\Sigma^{-1} P(T)$ can be shown not to do so. Essentially, Quillen's Lie algebra has an underlying chain complex that is an extension of ours by an acyclic complex-and the Lie algebra structure of the result contains the quadratic as well as the higher-degree information about a $\mathbb{Q}$-model.

3. The general case. In this section we will develop a theory of Q-models for non-split spaces using 1.10 through 1.20. We will first present the general obstruction theory and then apply it to a specific class of chain-complexes. Let $C$ be a $\mathbb{Q} \pi$-chain complex such that $H_{0}(C)=\mathbb{Q}, H_{1}(C)=0$. Then $C$ comes equipped with a unique chain-homotopy class of chain maps $C \rightarrow \widetilde{K(\pi, 1)}$. Let $g: C_{\mathfrak{B}} \rightarrow \mathfrak{B}(\pi)$ the associated chain-complex and map-see 1.15.

Consequently, we will assume given a chain-complex $C_{\mathfrak{B}}$ with a homomorphism $f: \mathrm{H}_{2}\left(C_{\mathfrak{B}}\right) \rightarrow I_{2}(\pi)$.

Claim. It suffices to build a $\mathbb{Q}$-model $M$ that is a twisted tensor product over $\mathfrak{B}(\pi)$ such that the underlying chain-complex of $M$ is chain-homotopy equivalent to $C_{\mathfrak{B}}$ via a map $h: C_{\mathfrak{B}} \rightarrow M$ such that the induced map $p_{*} \circ h_{*}: H_{2}\left(C_{\mathfrak{B}}\right) \rightarrow I_{2}(\pi)$ coincides with $f$.

This follows from an argument like that used in the proof of 2.7essentially we have to show that if there exists a map of DGA-coalgebras $A \rightarrow B$ satisfying the hypotheses of 1.19 then there exists (up to equivalence in the localized category $\mathrm{Ho}(\mathrm{DGC})$ ) a map of $\mathbb{Q}$ models with this property, and that that map may be assumed to be the projection of a twisted tensor product.

We will build a $\mathbb{Q}$-model for $C_{\mathfrak{B}}$-its $k$ th stage will be called $Y_{k}$. We begin with $Y_{0}=Y_{2}=\mathfrak{B}(\pi)$ and $g_{2}=$ identity map: $Y \rightarrow \mathfrak{B}(\pi)$. Let $(C)^{i}$ denote the $i$-skeleton of $C$ and let $h_{2}:\left(C_{\mathfrak{B}}\right)^{2} \rightarrow C\left(Y_{2}\right)$ be the map induced by $f$. In general $h_{k}:\left(C_{\mathfrak{B}}\right)^{k} \rightarrow C\left(Y_{k}\right)$ is a chain-map such that $\mathfrak{A}\left(h_{k}\right)$ is acyclic in dimensions $<k+1$ and $Y_{k}$ is a twisted tensor product over $Y_{k-1}$ (which makes all of the $Y_{k}$ twisted tensor products over $\mathfrak{B}(\pi)$ ). The construction of $Y$ proceeds in steps $A_{k}$ and $B_{k}$, like 
the main construction in [17]:

Step $A_{k}$. Extend $h_{k}$ to $\left(C_{\mathfrak{B}}\right)^{k+1}$ forming $h_{k+1}^{\prime}$. Strictly speaking this is not an extension since $h_{k}$ may be modified in dimension $k$ in the process. We require that $h_{k+1}^{\prime}\left|\left(C_{\mathfrak{B}}\right)^{k-1}=h_{k}\right|\left(C_{\mathfrak{B}}\right)^{k-1}$.

Step $B_{k}$. Form a twisted tensor product (by a $\left.\mathfrak{U}(M, k-1), Q\right)$ ) over $Y_{k}$ in such a way that $H_{k}\left(\mathfrak{A}\left(h_{k+1}^{\prime}\right)\right)$ is killed-the result is $Y_{k+1}$. Now lift $h_{k+1}^{\prime}$ to this twisted tensor product in a way that preserves the commutativity of the diagram above (up to a chain-homotopy) - this lift is $h_{k+1}$.

Claim. Step $B_{k}$ can always be carried out.

This follows by essentially the same argument that is used in [17]. If Step $A_{k}$ can be carried out $\mathfrak{A}\left(h_{k+1}^{\prime}\right)$ is acyclic below dimension $k$ of the evaluation map

$$
e: H^{k}\left(\mathfrak{A}\left(h_{k+1}^{\prime}\right), H_{k}\left(\mathfrak{A}\left(h_{k+1}^{\prime}\right)\right)\right) \rightarrow \operatorname{Hom}_{\mathbf{Z} \pi}\left(H_{k}\left(\mathfrak{A}\left(h_{k+1}^{\prime}\right)\right), H_{k}\left(\mathfrak{A}\left(h_{k+1}^{\prime}\right)\right)\right)
$$

is an isomorphism. This implies that there exists an element $a \in$ $H^{k}\left(Y_{k}, H_{k}\left(\mathfrak{A}\left(h_{k+1}^{\prime}\right)\right)\right)$ such that the homomorphism $H_{k}\left(Y_{k}\right) \rightarrow$ $H_{k}\left(\mathfrak{A}\left(h_{k+1}^{\prime}\right)\right)$ induced by evaluation coincides with that induced by the inclusion $C\left(Y_{k}\right) \rightarrow \mathfrak{A}\left(h_{k+1}^{\prime}\right)$. Choose a chain-map $c: C\left(Y_{k}\right) \rightarrow$ $\Sigma \mathfrak{U}\left(\mathfrak{A}\left(h_{k+1}^{\prime}\right), k, Q\right)$ that induces an isomorphism in homology in dimension $k+1$. Proposition 2.3 implies that we can convert $c$ into a twisting cochain, $c^{\prime}$. Now we form the twisted tensor product $Y_{k+1}$ via $c^{\prime}$. The arguments in 2.4 and 2.5 of [17] then imply:

A. There exists a unique chain-homotopy class of lifts of $h_{k+1}^{\prime}$ to $C\left(Y_{k+1}\right)$ making the diagram above commute;

B. That lift, $h_{k+1}$, has the property that $H_{i}\left(\left(\mathfrak{A}\left(h_{k+1}\right)\right)=0\right.$ for $i \leq k$.

It follows that the only significant obstruction we will encounter involves carrying out Step $A_{k}$. The obstructions to doing this can be characterized in terms of elementary homological algebra, as was done in [17].

Definition 3.1. Let $h_{k},\left(C_{\mathfrak{B}}\right)^{k+1}$ be as in step $k$ described above. Define a class (which will be called the $k$ th obstruction to finding a Q-model for $C) c_{k} \in H^{k+1}\left(C_{\mathfrak{B}} ; H_{k}\left(\mathfrak{A}\left(g_{k}\right)\right)\right)$ as follows:

(a) Note that, since $h_{k}$ is a chain map, the cycle submodule of $\left(C_{\mathfrak{B}}\right)_{k}$ is mapped into the cycle submodule of $\left(\mathfrak{A}\left(g_{k}\right)\right)_{k}$ so that we get a map from the cycle submodule of $\left(C_{\mathfrak{B}}\right)_{k}$ to $H_{k}\left(\mathfrak{A}\left(g_{k}\right)\right)$. 
(b) Consider the composite $\left(C_{\mathfrak{B}}\right)_{k+1} \rightarrow\left(C_{\mathfrak{B}}\right)_{k} \rightarrow H_{k}\left(\mathfrak{A}\left(g_{k}\right)\right)$, where the map on the left is the boundary map of $T$ and that on the right is induced by $h_{k}$. This composite defines a cocycle that gives the class $c_{k}$.

In the remainder of this section we will apply this obstruction theory to some problems. The first step is the computation of a $\mathbb{Q}$-model for $\mathfrak{B}(\pi)$. I feel that this may be a difficult problem in general but something can be said in the case where the homological dimension of $\mathfrak{B}(\pi)$ is limited (note that this is essentially the same as the homological dimension of the group $\pi$ ).

Proposition 3.2. If the homological dimension of the group $\pi$ is $\leq 5$ then a $\mathbb{Q}$-model for $\mathfrak{B}(\pi)$ is determined by the class of

$$
\operatorname{Ext}_{\mathbb{Q} \pi}^{2}\left(I_{2}(\pi), I_{2}(\pi) \otimes I_{2}(\pi)\right)
$$

defined by the induced coproduct, $\Delta$, of $\mathfrak{B}(\pi)$.

Remarks. 1. The coproduct, $\Delta$, is derived from the geometric coproduct of $K(\pi, 1)$.

2. Here $I_{2}(\pi)$ is $H_{2}(\mathfrak{B}(\pi))=$ kernel $\mathfrak{B}(\pi)_{2} \stackrel{\partial}{\rightarrow} \mathfrak{B}(\pi)_{1}$. The class of $\operatorname{Ext}_{\mathbb{Q} \pi}^{2}\left(I_{2}(\pi), I_{2}(\pi) \otimes I_{2}(\pi)\right)$ in quesiton is that induced by $\Delta: \mathfrak{B}(\pi)_{4} \rightarrow$ $\mathfrak{B}(\pi)_{2} \otimes \mathfrak{B}(\pi)_{2}$-it is well known that the chain-homotopy class of the chain-map $\Delta$ is characterized by this element of the Ext-group.

Proof. Consider the steps involved in building a $\mathbb{Q}$-model for $\mathfrak{B}(\pi)$. We will clearly have to begin with a $\mathfrak{U}\left(I_{2}(\pi), 2, Q\right)$. The next stage requires a factor of $\mathfrak{U}\left(\Gamma\left(I_{2}(\pi)\right), 3, Q\right)$ to kill off the homology of $\mathfrak{U}\left(I_{2}(\pi), 2, Q\right)$ in dimension 4 . Here $\Gamma\left(I_{2}(\pi)\right)$ is the Whitehead quadratic functor of $I_{2}(\pi)$-since we are working over $\mathbb{Q}$ it is isomorphic to $I_{2}(\pi) \otimes I_{2}(\pi)$. In order to see how to select the class of the twisting cocycle used to form this stage note that

$$
\begin{aligned}
& H^{4}\left(\mathfrak{U}\left(I_{2}(\pi), 2, Q\right), I_{2}(\pi) \otimes I_{2}(\pi)\right) \\
& \quad=\operatorname{Ext}_{\mathbb{Q} \pi}^{2}\left(I_{2}(\pi), I_{2}(\pi) \otimes I_{2}(\pi)\right) \\
& \quad \oplus \operatorname{Hom}_{\mathbb{Z} \pi}\left(I_{2}(\pi) \otimes I_{2}(\pi), I_{2}(\pi) \otimes I_{2}(\pi)\right) .
\end{aligned}
$$

Since we want to kill off the homology of $\mathfrak{U}\left(I_{2}(\pi), 2, Q\right)$ in dimension 4 we will clearly want to select a class whose $\operatorname{Hom}_{\mathbf{Z} \pi}\left(I_{2}(\pi) \otimes\right.$ $I_{2}(\pi), I_{2}(\pi) \otimes I_{2}(\pi)$ )-factor is an isomorphism of $I_{2}(\pi) \otimes I_{2}(\pi)$. We also want the chain-homotopy class of the coproduct of the $\mathbb{Q}$-model 
to agree with that of $\mathfrak{B}(\pi)$. Let $P \subset \mathfrak{U}\left(I_{2}(\pi), 2, Q\right)$ be a suspended projective resolution of $I_{2}(\pi)$. It is a direct summand. Let $\alpha: \mathfrak{B}(\pi) \rightarrow P$ be a chain-homotopy equivalence-the chain-homotopy class of $\alpha$ is unique-and let $\beta: P \rightarrow \mathfrak{B}(\pi)$ be a chain-homotopy inverse. Let $c \in \operatorname{Ext}_{\mathfrak{Q} \pi}^{2}\left(I_{2}(\pi), I_{2}(\pi) \otimes I_{2}(\pi)\right)$ denote the class of $\Delta: \mathfrak{B}(\pi)_{4} \rightarrow \mathfrak{B}(\pi)_{2} \otimes$ $\mathfrak{B}(\pi)_{2}$.

Claim. The $\operatorname{Ext}_{\mathrm{Q} \pi}^{2}\left(I_{2}(\pi), I_{2}(\pi) \otimes I_{2}(\pi)\right)$-factor of the twisting cochain must agree with $(\alpha \otimes \alpha)^{*} c \beta^{*}$. Note that the following diagram

$$
\begin{aligned}
& \mathfrak{U}\left(I_{2}(\pi), 2, Q\right)_{4}=P_{4} \oplus P_{2} \otimes P_{2} \stackrel{D}{\longrightarrow} \quad P_{2} \otimes P_{2} \\
& \alpha \oplus \alpha \otimes \alpha \cdot c \uparrow \quad \uparrow \alpha \otimes \alpha \\
& \mathfrak{B}(\pi) \stackrel{\Delta}{\longrightarrow} \mathfrak{B}(\pi) \otimes \mathfrak{B}(\pi)
\end{aligned}
$$

commutes in dimension 4-here $D$ is the coproduct of $\mathfrak{U}\left(I_{2}(\pi), 2, Q\right)$. The vertical map on the left side will only be a chain-map (at least up to dimension 5) into a twisted tensor product if the twisting cochain is essentially $c \cdot \beta$. This implies the claim.

The remainder of the result follows from the fact that $\pi$ is of homological dimension $\leq 5$. This implies that varying the twisting cochains used to build the $\mathbb{Q}$-model for $\mathfrak{B}(\pi)$ in higher dimensions (and killing off the homology above dimension 2) will only alter the resulting $\mathbb{Q}$ model by an isomorphism of DGA-coalgebras-see B.15 in Appen$\operatorname{dix} B$.

Proposition 3.3. Let $\pi$ be a group of $\mathbb{Q}$-homological dimension $\leq$ 5 and let $C$ be any projective $\mathbb{Q} \pi$-chain complex. Then there exists at most one obstruction to topologically realizing $C$ : it is $\imath^{*}(\alpha \otimes v) \in$ $H^{5}\left(C ; H_{2}(C) \otimes I_{2}(\pi)\right)$, where

1. $\alpha \in H^{3}\left(\pi, H_{2}(C)\right)$ is the first homological $k$-invariant;

2. $\imath: C_{\mathfrak{B}} \rightarrow \mathfrak{B}(\pi)$ is the projection;

3. $v: \mathfrak{B}(\pi)_{2} \rightarrow I_{2}(\pi)$ maps elements to their homology classes.

REMARKS. 1. The last statement uses the fact that $\mathfrak{B}(\pi)_{5}=\mathfrak{B}(\pi)_{3} \otimes$ $\mathfrak{B}(\pi)_{2}$-at least for a $\mathbb{Q}$-model for $\mathfrak{B}(\pi)$. By abuse of notation we are using $\mathfrak{B}(\pi)$ to denote a $\mathbb{Q}$-model for $\mathfrak{B}(\pi)$.

2. $\alpha \otimes v$ denotes the external product of cohomology classes.

Proof. We will build a candidate for a $\mathbb{Q}$-model for $C_{\mathfrak{B}}$. Let $C^{+}$ denote a desuspension of the algebraic mapping cone of $C \rightarrow \overparen{K(\pi, 1)}$. Then $C$ is chain-homotopy equivalent to a twisted direct sum 
$\widetilde{K(\pi, 1)} \oplus_{\alpha} C^{+}$and $C_{\mathfrak{B}}$ is chain-homotopy equivalent to the corresponding twisted direct sum $\mathfrak{B}(\pi) \oplus_{\alpha} C^{+}$-see B.4 and 1.15. Our candidate for a $\mathbb{Q}$-model for $C_{\mathfrak{B}}$ is derived from $\mathfrak{B}(\pi) \otimes_{t\left(\alpha^{\prime}\right)} S\left(C^{+} \otimes Q\right)$ see B.7 for the notation $t\left(\alpha^{\prime}\right)$. Here $\alpha^{\prime}: \mathfrak{B}(\pi) \rightarrow \Sigma C^{+} \otimes Q$ is a lifting of $\alpha$.

Note that $C^{+} \otimes Q$ is chain-homotopy equivalent to $C^{+}$and can be used as the first step in building a $Q$-model for $C^{+} \otimes Q$ since it contains an imbedded subcomplex isomorphic to $C^{+} \otimes Q$ with the property that the coproduct is trivial on this subcomplex. This means that we can use the obstruction theory developed in this section to kill off $S\left(C^{+} \otimes Q\right) / C^{+} \otimes Q$. After each step the vanishing of the coproduct on the imbedded copy of $C^{+} \otimes Q$ means that the twisted tensor product used will be trivial on $C^{+} \otimes Q$ and the result will still contain an imbedded copy of $C^{+} \otimes Q$.

In our case we have $\mathfrak{B}(\pi) \otimes_{t\left(\alpha^{\prime}\right)} S\left(C^{+} \otimes Q\right)$. This contains an imbedded copy of $C^{+} \otimes Q$ as a subcomplex and an imbedded copy of $\mathfrak{B}(\pi) \oplus_{\alpha^{\prime}} C^{+} \otimes Q$ - the latter is not a subcomplex, however. In applying our obstruction theory we will map this copy of $\mathfrak{B}(\pi) \oplus_{\alpha^{\prime}} C^{+} \otimes Q$ to its image in $\mathfrak{B}(\pi) \otimes_{t\left(\alpha^{\prime}\right)} S\left(C^{+} \otimes Q\right)$ and compute the obstruction to the map being a chain-map. The boundary in the twisted tensor product is $\partial_{\mathfrak{B}(\pi)}+\partial_{S\left(C^{+} \otimes Q\right)}+\alpha \cap *$, and in the twisted direct sum it is $\partial_{\mathfrak{B}(\pi)}+\partial_{C^{+} \otimes Q}+\alpha$. The only possible contribution to the obstruction can occur where these two boundaries differ (since the obstructions result from computing the homology images of $\partial_{\mathfrak{B}(\pi) \oplus_{n} C^{+} \otimes Q}$-which will be 0 if that boundary agrees with the boundary of the twisted tensor product). The lowest dimension in which this can happen is 5-the difference is equal to the class of the cocycle

$$
\mathfrak{B}(\pi)_{5} \oplus C^{+} \otimes Q \stackrel{l}{\rightarrow} \mathfrak{B}(\pi)_{5} \stackrel{\Delta}{\rightarrow} \mathfrak{B}(\pi)_{3} \otimes \mathfrak{B}(\pi)_{2} \stackrel{\alpha \otimes v}{\rightarrow} H_{2}(C) \otimes I_{2}(\pi) .
$$

Note that, since the obstruction restricts to 0 on the $C^{+} \otimes Q$ summand it is not necessary to ever modify the inclusion on the $C^{+} \otimes Q$ summand to make it a chain-map. It follows that, if this obstruction vanishes, we don't create any additional obstructions in higher dimensions by modifying the inclusion to make it a chain-map ${ }^{5}$.

A similar argument implies that:

COROLlaRY 3.4. If the $\mathbb{Q}$-homological dimension of $\pi$ is $k>2$ there exist at most $k-4$ significant obstructions to topologically realizing a $\mathbb{Q} \pi$-chain complex.

\footnotetext{
${ }^{5}$ This might have happened if we had to alter the inclusion of $C^{+} \oplus Q$ into $S\left(C^{+} \oplus Q\right)$.
} 
The remainder of this section will be spent showing that the obstruction theory is nontrivial. Let $\pi=\mathbb{Z}^{5}$ and let $C=Q \oplus_{\alpha} \Sigma^{2} Q$, where $\alpha \in H^{3}(\pi, \mathbb{Q})$ is any nontrivial class. Then the obstruction to realizing $C$ is essentially $\imath^{*}(\alpha \otimes v) \in H^{5}\left(C ; \mathbb{Q} \otimes I_{2}(\pi)\right)=H^{5}\left(C ; I_{2}(\pi)\right)=$ $H^{5}\left(\pi ; I_{2}(\pi)\right) \oplus H^{3}\left(\pi ; I_{2}(\pi)\right)$. The obstruction is contained in the $H^{5}\left(\pi ; I_{2}(\pi)\right)$-summand and we will show that its image in $H^{5}(\pi ; \mathbb{Q})$ $=\mathbb{Q}$ under the change of coefficient map is nontrivial. The rational cohomology algebra of $\mathbb{Z}^{5}$ is the rank-5 exterior algebra. The class $v$ maps to the class of $H^{2}(\pi ; \mathbb{Q})$ that maps each generator to 1 , and it isn't hard to see that the cup-product of this class with a nonzero class of $H^{3}(\pi, \mathbb{Q})$ will be nonzero.

Appendix A. In this appendix we will define appropriate homotopy theories on the categories in the diagram in the beginning of $\S 1$ and show that the functors between them preserve homotopy theories. We will make extensive use of the results and methods of Quillen in part II of [11]. In fact, in most cases it will only be necessary to indicate a few minor ways our arguments differ from Quillen's.

Cofibrations in $\mathfrak{S}_{2}-\pi$ are defined to be inclusions of simplicial sets equipped with a $\pi$-action (i.e. the inclusion preserves the free $\pi$-action above dimension 0 ). Here objects of $\mathfrak{S}_{2}-\pi$ are regarded as simplicial sets where simplices above dimension 0 are indexed by elements of $\pi$. Fibrations in $\mathfrak{S}_{2}-\pi$ are defined to be maps $f$ such that $|\mathfrak{F}(f)| / \pi$ is a fibration of topological spaces. Note that of trivial cofibrations can be lifted by lifting simplices $1 \cdot \sigma$ (in the indexing scheme) and translating by the action of $\pi$.

In SGp- $\pi$ fibrations and cofibrations are defined exactly as in [11] except that cofibrations are retracts of free maps in which the free group that is being attached has its free generators indexed by $\pi$.

In SCHA- $\pi$ and SLA- $\pi$ fibrations and cofibrations of simplicial Hopf algebras are defined as in [11] except that the term "simplicial vector spaces"6 is replaced by "simplicial projective $\mathbb{Q} \pi$-modules".

In DGL- $\pi$ a fibration is a surjective map and a cofibration $A \rightarrow B$ is a retract of a map $A \rightarrow A \oplus D$ where $D$ is a free $\pi$-Lie algebra on a free $\mathbb{Q} \pi$-module. Here, a free $\pi$-Lie algebra $D$ is a free Lie algebra subject to the condition that $[q \cdot a, q \cdot b]=q \cdot[a, b]$ for all $q \in \pi$ and $a, b \in D$. In DGC- $\pi$ fibrations and cofibrations are defined exactly as in part II of [11]. For that to make sense we need only verify that the appropriate version of Lemma 5.6 in [11] holds. But this follows from

\footnotetext{
${ }^{6}$ Applied to the primitive subcomplexes in SCHA- $\pi$.
} 
the fact that the underlying vector spaces of the Lie algebras involved are projective $\mathbb{Q} \pi$-modules.

Appendix B-Algebraic results. We will $f i x$ a projective resolution $Q$ of $\mathbb{Q}$ over $\mathbb{Q} \pi$.

Definition B.1. Let $M$ be a $\mathbb{Q} \pi$-module. Then $M_{*}$ represents the projective $\mathbb{Q} \pi$-resolution of $M$ that results from forming the tensor product $M \otimes Q$ and equipping it with the diagonal $\pi$-action.

REMARK. We will also use $M_{i}$ to refer to $M \otimes Q_{i}$.

Definition B.2. If $f: C \rightarrow D$ is a chain-map the algebraic mapping cone of $f, \mathfrak{A}(f)$, is defined to have chain-modules $\mathfrak{A}(f)_{i+1}=C_{i} \oplus D_{i+1}$ and boundary

$$
\left(\begin{array}{cc}
d_{C} & 0 \\
(-1)^{i} f & d_{D}
\end{array}\right): C_{i} \oplus D_{i+1} \rightarrow C_{i-1} \oplus D_{i}
$$

We will use the following elementary property of algebraic mapping cones:

Proposition B.3. Suppose the maps $f, f^{\prime}$ and $s$ in the following diagram

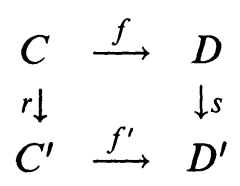

are defined in all dimensions and are chain maps. Then there exists a chain-map $r$ defined up to a dimension $k$ such that $s \circ f-f^{\prime} \circ r=$ $d \Phi: C \rightarrow D^{\prime}$ if and only if there exists a map $\mathfrak{m}: \mathfrak{A}(f) \rightarrow \mathfrak{A}\left(f^{\prime}\right)$ that is: (i) defined up to dimension $k+1$ and is a chain-map; (ii) makes the following commute:

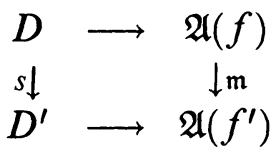

up to dimension $k$.

Proof. Suppose that $r$ exists with the stated properties. Then

$$
\mathfrak{m}=\left(\begin{array}{cc}
r & 0 \\
(-1)^{i} \Phi & s
\end{array}\right): C_{i+1} \oplus D_{i} \rightarrow C_{i+1}^{\prime} \oplus D_{i}^{\prime}
$$

is easily verified to be a chain-map, where $i \leq k$. If $\mathfrak{m}$ exists with the stated properties the last diagram in the statement of the propo- 
sition implies that $\mathfrak{m}$ induces a chain-map via the projections $p: \mathfrak{A}(f) \rightarrow C$.

Definition B.4. Let $f: C \rightarrow \Sigma D$ be a chain-map. Then the twisted direct sum of $C$ and $D$ with respect to $f$ is defined to be $\Sigma^{-1} \mathfrak{A}(f)$.

Definition B.5. Let $B$ be a DGA-coalgebra and $A$ be a DGAalgebra. Let $x$ and $y$ be chain maps from $B$ to $A$. Then:

1. the cap product with respect to $x$, denoted $x \cap$, is defined to be the composite $\left(1_{B} \otimes \varphi\right) \circ\left(1_{B} \otimes x \otimes 1_{A}\right) \circ\left(\Delta \otimes 1_{A}\right): B \otimes A \rightarrow B \otimes A$;

2. the cup product of $x$ and $y$, denoted $x \cup y$, is the composite $\mu \circ(x \otimes y) \circ \Delta: B \rightarrow A$;

3. if $x$ is a map of degree -1 that has the property that $d x+x \cup x=0$ then the twisted tensor product $B \otimes_{x} A$ is defined to be the chaincomplex $B \otimes A$, equipped with the differential $d_{x}=d_{B \otimes A}+x \cap$.

Remarks. 1. The condition on $x$ in statement 3 implies that the differential for the twisted tensor product is self-annihilating-see [10]. In this case the map $x$ is called the twisting cochain of the twisted tensor product.

2. A quadruple $(A, \mu, \psi)$ will be called a DGA-Hopf algebra if $(A, \mu)$ is a DGA-algebra, $(A, \psi)$ is a DGA-coalgebra, and $\psi: A \rightarrow A \otimes A$ is a homomorphism of DGA-algebras or dually $\mu: A \otimes A \rightarrow A$ is a homomorphism of DGA-coalgebras.

3. Twisted tensor products were originally defined to study the chain complex of a fibration. Fibrations can be described as semisimplicial complexes as "twisted cartesian products"-see [9, p. 405] or [14, Chapter 2]. The main result (see [9] for details) in this direction is that there exists a chain-homotopy euqivalence $\left(f_{\xi}, g_{\xi}, \varphi_{\xi}\right)$ : $C\left(B \times \times_{\xi} F\right) \rightarrow C(B) \otimes_{\xi} C(F)$ from the chain complex of the total space of a fibration to the twisted tensor product of that of the base and that of the fiber.

3. The Koszul convention (see [8] for a description of this convention) implies that if $\Delta(x)=\sum_{i} x_{i} \otimes y_{i}$, then

$$
(\alpha \cup \beta)=\sum_{i}(-1)^{\operatorname{deg}(\beta) \cdot \operatorname{dim}\left(x_{i}\right)} \alpha\left(x_{i}\right) \cdot \beta\left(y_{i}\right)
$$

and

$$
\alpha \cap(x \otimes y)=\sum_{i}(-1)^{\operatorname{deg}(\alpha) \cdot \operatorname{dim}\left(x_{i}\right)} x_{i} \otimes \alpha\left(y_{i}\right) \cdot y_{i}
$$


In addition the following statements are well known:

Facts B.6. (a) $d(\alpha \cup \beta)=(d \alpha) \cup \beta+(-1)^{\operatorname{deg}(\alpha)} \alpha \cup(d \beta)$; and

(b) $d(\alpha \cap x)=(d \alpha) \cap x+(-1)^{\operatorname{deg}(\alpha)} \alpha \cap(d x)$.

Proposition B.7. Let $C$ be a commutative DGA-coalgebra A a commutative DGA-algebra and let $\alpha: C \rightarrow \Sigma A$ be a chain-map. Let $t\left(\alpha^{\prime}\right)$ : $C \rightarrow A$ be defined by $t\left(\alpha^{\prime}\right)(x)=(-1)^{\operatorname{dim}(x)-1} \alpha(x)$. Then $t\left(\alpha^{\prime}\right): C \rightarrow A$ defines a twisting cochain.

REMARKS. 1. Since $\alpha$ is a chain-map $d \alpha^{\prime}=0$. We must show that $\alpha^{\prime} \cup \alpha^{\prime}=0$ - see the definition of a twisting cochain in the remarks following B.5. This follows from the fact that $C$ is commutativeand $A$ is commutative with the opposite parity-because of the shift in dimensions. If $e$ is of dimension $2 n+2$ then interchanging the factors of $\Delta(e)$ multiplies $\Delta(e)$ by $(-1)^{n+1}$, whereas interchanging two factors in $A$ multiplies their product by $(-1)^{n}$.

2. A similar argument implies that, if the differential of $A$ is identically zero, any cocycle $\alpha: C \rightarrow A$ (in some dimension) defines a twisting cochain.

Definition B.8. Let $C$ be a $\mathbb{Q} \pi$-chain-complex. Then the symmetric algebra $S(C)$ is defined to be $T(C) / I$, where $T(C)$ is the tensor algebra and $I$ is the ideal generated by elements of the form $\{e \otimes f-$ $\left.(-1)^{i j} f \otimes e\right\}$ where $e \in C_{i}$ and $f \in C_{j}$.

Remarks. 1. $S(C)$ has the structure of a DGA-Hopf algebra. It inherits its coalgebra structure from $T(C)$ and its algebra structure is induced by tensor-product multiplication. $U(M, n) \cong S\left(\Sigma^{n} M\right)$, where the isomorphism maps $\gamma_{i}(x)$ to $x \otimes \cdots \otimes x(i$ copies of $x)$ in the evendimensional case.

2. It is well-known that $S(C \oplus D) \cong S(C) \otimes S(D)$. It is possible to show that $S(*)$ maps twisted direct sums into twisted tensor products:

Proposition B.9. Let $C \oplus_{f} D$ be a twisted direct sum (see B.4). Then $S\left(C \oplus_{f} D\right) \cong S(C) \otimes_{f^{\prime}} S(D)$, where $f^{\prime}$ is the twisting cochain equal to $t(f)$ on $C$ in $S(C)$ and zero on other elements of $S(C)$.

Proof. We use the natural isomorphism $S(C \oplus D) \cong S(C) \otimes S(D)$ described above. First we recall the concept of a principal twisted object, defined by V. K. A. M. Gugenheim in [9, §2]. 
Definition. Let $B$ be a DGA-coalgebra and let $A$ be a DGAalgebra. A principal twisted object is a left $B$-comodule and right $A$-module that is $B \otimes A$, apart from the differentials.

REMARK. The important property of a principal twisted object, for our purposes, is that it must be a twisted tensor product-see [9, Proposition 2.2].

Claim. $S\left(C \oplus_{f} D\right)$ is a principal twisted object. To prove this we must show a natural structure as a DGA-comodule over $S(C)$ and a DGA-module over $S(D)$. But these facts follow from the fact that $D$ is a sub-chain-complex of $C \oplus_{f} D$ and there exists a natural projection $C \oplus_{f} D \rightarrow C$. We define the right action of $S(D)$ on $S\left(C \oplus_{f} D\right)$ by composing $1 \otimes S(i): S\left(C \oplus_{f} D\right) \otimes S(D) \rightarrow S\left(C \oplus_{f} D\right) \otimes S\left(C \oplus_{f} D\right)$ with the multiplication map $S\left(C \oplus_{f} D\right) \otimes S\left(C \oplus_{f} D\right) \rightarrow S\left(C \oplus_{f} D\right)$. The comodule structure is defined by composing the coproduct map $S\left(C \oplus_{f} D\right) \rightarrow S\left(C \oplus_{f} D\right) \otimes S\left(C \oplus_{f} D\right)$ with $S(p) \otimes 1: S\left(C \oplus_{f} D\right) \otimes$ $S\left(C \oplus_{f} D\right) \rightarrow S(C) \otimes S\left(C \oplus_{f} D\right)$. It is not hard to see that the isomorphism $S(C \oplus D) \cong S(C) \otimes S(D)$ preserves these structures and the formula for the twisting cochain follows.

Proposition B.10. Let $(C, \psi, \varepsilon)$ be a DGA-coalgebra and let $\left(D, \psi^{\prime}, \mu, \varepsilon^{\prime}\right)$ be a DGA-Hopf algebra, where the $\psi$ 's are the coproducts, the $\varepsilon$ 's are the augmentations, and $\mu$ is the multiplication of $D$. Form the twisted tensor product $C \otimes_{x} D$ with respect to some twisting cochain $x: C \rightarrow D$. Then the tensor product coproduct on $C \otimes_{x} D$ is a chain-map if and only if $x$ has the property that, for every $c \in C$, $\psi^{\prime}(x(c))=1 \otimes x(c)+x(c) \otimes 1 \in D \otimes D$.

Remarks. 1. A twisted tensor product that satisfies the hypotheses of this proposition will be said to support the untwisted coproduct structure. In general, a twisted tensor product like that in the statement will have a coproduct that is twisted in some sense.

2. The hypotheses imply that, in some sense, the restriction of the coproduct of $D$ to the image of $x$ is trivial.

Proof. We note the existence of an isomorphism of chain-complexes $1_{C} \otimes T \otimes 1_{D}:(C \otimes C) \otimes_{\varepsilon \otimes x+x \otimes \varepsilon}(D \otimes D) \rightarrow\left(C \otimes_{x} D\right) \otimes\left(C \otimes_{x} D\right)$. The conclusion now follows from the observation that $\psi \otimes \psi^{\prime}: C \otimes_{x} D \rightarrow$ $(C \otimes C) \otimes_{\varepsilon \otimes x+x \otimes \varepsilon}(D \otimes D)$ is a chain-map, by the hypotheses on $x$.

The following is similar. 
Proposition B.11 Let $C \otimes_{x} A$ be a twisted tensor product. If the twisting cochain $x$ has the property that $x\left(c_{1} \cdot c_{2}\right)=0, c_{i} \in C$, whenever $c_{1}$ and $c_{2}$ have augmentation 0 then $C \otimes_{x} A$ has the untwisted product structure.

Proof. We must essentially show that the map $\mu_{C} \otimes \mu_{A}:(C \times C) \otimes_{y}$ $(A \otimes A) \rightarrow C \otimes_{x} A$ is a chain-map, where $y$ is the twisting cochain $x \otimes \eta+\eta \otimes x$. Here $\eta$ is the composite of the augmentation of $C$ with the splitting $\mathbf{Z} \rightarrow A_{0}$ of the augmentation of $A$ that carries 1 to the identity of $A$. But this follows immediately from the hypotheses.

Suppose that $C$ is a DGA-coalgebra and $f: C \rightarrow \Sigma^{n+1} M_{*}$ is a chainmap. Then $f^{\prime}$ (see B.7) defines a twisting cochain $t(f): C \rightarrow \mathfrak{U}(M, n, Q)$, where the image of $f^{\prime}\left(C_{n+i+1}\right)$ is in $\cdots \otimes \mathbb{Q} \otimes \cdots U\left(M_{i}, n+i\right) \cdots \otimes \mathbb{Q} \otimes$ and the twisted tensor product $C \otimes_{t(f)} \mathfrak{U}(M, n, Q)$ supports the untwisted coproduct structure. This follows from B.7 and B.10.

Definition B.12. Suppose that $X$ is a DGA-coalgebra and $F$ is a DGA-algebra. Two twisting cochains $\xi_{i}: X \rightarrow F, i=1,2$ will be called $\xi$-cobordant if there exists a twisting cochain $\Xi: X \otimes I \rightarrow F$ such that $\Xi \mid X \otimes p_{i}=\xi$.

Remarks. 1. Recall that the unit interval $I$ is defined, as a DGAcoalgebra, by:

(a) $I_{0}=\mathbb{Z} \oplus \mathbb{Z}$, generated by $p_{0}$ and $p_{1}$;

(b) $I_{1}=\mathbb{Z}$, generated by $q$;

(c) $\Delta q=p_{1}-p_{0}$;

(d) $\Delta p_{i}=p_{i} \otimes p_{i}, \Delta q=q \otimes p_{0}+p_{1} \otimes q$;

2. The twisting cochain $\Xi: X \otimes I \rightarrow F$ will be called the cobordism between the $\xi_{i}$.

Proposition B.13 Let $\xi, \eta$ be $\xi$-cobordant twisting cochains from $X$ to $F$ and let $\Xi: X \otimes I \rightarrow F$ be the cobordism between them. Then there exists an isomorphism $v: X \otimes_{\xi} F \rightarrow X \otimes_{\eta} F$ such that the following diagram commutes:

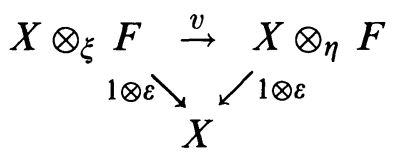

where $\varepsilon: F \rightarrow \mathbb{Q}$ is the augmentation. In addition the following map is an isomorphism of chain-complexes: $V:(X \otimes I) \otimes_{\xi \otimes I} F \rightarrow(X \otimes I) \otimes_{\Xi}$ $F$, where $V \mid X \otimes p_{0} \otimes F$ and $V \mid X \otimes q \otimes F$ is the identity map, and $V \mid X \otimes p_{1} \otimes F$ is $v$. 
REMARKs. The isomorphism also turns out to be a homomorphism of comodules over $\Delta$, where $\Delta$ is the comultiplication of $X$. In addition, if $F$ is a $D G A$-algebra then $v$ is a right $F$-module homomorphism.

Proof. Define the map $\mathscr{I}: X \rightarrow X \otimes I$ to send $x \in X$ to $(-1)^{\operatorname{dim}(x)} x \otimes$ $q \in X \otimes I$. We will define $v$ to be $1+(\Xi \circ \mathscr{I}) \cap^{*}: X \otimes_{\xi} F \rightarrow X \otimes_{\eta} F$. In order to prove that this has the required properties we must prove the following identity:

Fact 1. $(\Xi \cup \Xi) \circ \mathscr{I}=\eta \cup(\Xi \circ \mathscr{I}) \cup \xi$.

Proof of identity. This follows from the definition of the coproduct on $I: \Delta(x \otimes q)=(1 \otimes T \otimes 1) \circ \Delta(x) \otimes\left(q \otimes p_{0}+p_{1} \otimes q\right)$ so

$$
\Delta \circ \mathscr{I}(x)=(-1)^{\operatorname{dim}(x)}(1 \otimes T \otimes 1) \circ \Delta(x) \otimes\left(q \otimes p_{0}+p_{1} \otimes q\right) .
$$

Let $\Delta(x)=\sum_{i} x_{i} \otimes_{i} x$ and let $d(i)=\operatorname{dim}\left(x_{i}\right)$, so $\operatorname{dim}\left({ }_{i} x\right)=\operatorname{dim}(x)-$ $d(i)$. Then

$$
\begin{aligned}
\Delta \circ \mathscr{I}(x)= & (-1)^{\operatorname{dim}(x)}(1 \otimes T \otimes 1) \circ \sum_{i} x_{i} \otimes{ }_{i} x \otimes q \otimes p_{0} \\
& +(-1)^{\operatorname{dim}(x)}(1 \otimes T \otimes 1) \circ \sum_{i} x_{i} \otimes{ }_{i} x \otimes p_{1} \otimes q \\
= & (-1)^{\operatorname{dim}(x)+\operatorname{dim}(x)} \sum_{i}(-1)^{d(i)} x_{i} \otimes q \otimes{ }_{i} x \otimes p_{0} \\
& +(-1)^{\operatorname{dim}(x)} \sum_{i} x_{i} \otimes p_{1} \otimes{ }_{i} x \otimes q \\
= & \sum_{i}(-1)^{d(i)} x_{i} \otimes q \otimes{ }_{i} x \otimes p_{0} \\
& +(-1)^{\operatorname{dim}(x)} \sum_{i} x_{i} \otimes p_{1} \otimes{ }_{i} x \otimes q,
\end{aligned}
$$

where the $(-1)^{\operatorname{dim}(x)-d(i)}$ results from the operator $T$ and the fact that $q$ is 1-dimensional. If we plug this into $\Xi \otimes \Xi$ we get

$$
\begin{aligned}
(\Xi \otimes \Xi) & \circ \sum_{i}(-1)^{d(i)} x_{i} \otimes q \otimes{ }_{i} x \otimes p_{0} \\
& +(\Xi \otimes \Xi) \circ(-1)^{\operatorname{dim}(x)} \sum_{i} x_{i} \otimes p_{1} \otimes{ }_{i} x \otimes q \\
= & \sum_{i}(-1)^{d(i)}(-1)^{d(i)+1} \Xi\left(x_{i} \otimes q\right) \otimes \Xi\left({ }_{i} x \otimes p_{0}\right) \\
& +(-1)^{\operatorname{dim}(x)} \sum_{i}(-1)^{d(i)} \Xi\left(x_{i} \otimes p_{1}\right) \otimes \Xi\left({ }_{i} x \otimes q\right)
\end{aligned}
$$


(where the additional signs result from our convention regarding the evaluation of tensor products of maps on tensor products of elements here we use the fact that the degree of $\Xi$ is -1 )

$$
\begin{aligned}
=- & \sum_{i} \Xi\left(x_{i} \otimes q\right) \otimes \Xi\left({ }_{i} x \otimes p_{0}\right) \\
& +(-1)^{\operatorname{dim}(x)} \sum_{i}(-1)^{d(i)} \Xi\left(x_{i} \otimes p_{1}\right) \otimes \Xi\left({ }_{i} x \otimes q\right) \\
=- & \sum_{i}(-1)^{d(i)} \Xi \circ \mathscr{I}\left(x_{i}\right) \otimes \Xi\left({ }_{i} x \otimes p_{0}\right) \\
& +\sum_{i}(-1)^{\operatorname{dim}(x)+d(i)+\operatorname{dim}(x)-d(i)} \Xi\left(x_{i} \otimes p_{1}\right) \otimes \Xi \circ \mathscr{I}\left({ }_{i} x\right) \\
=- & \sum_{i}(-1)^{d(i)} \Xi \circ \mathscr{I}\left(x_{i}\right) \otimes \Xi\left({ }_{i} x \otimes p_{0}\right) \\
& +\sum_{i} \Xi\left(x_{i} \otimes p_{1}\right) \otimes \Xi \circ \mathscr{I}\left({ }_{i} x\right) \\
=- & (\Xi \circ \mathscr{I} \otimes \xi) \circ \sum_{i} x_{i} \otimes{ }_{i} x+(\eta \otimes \Xi \circ \mathscr{I}) \circ \sum_{i} x_{i} \otimes{ }_{i} x .
\end{aligned}
$$

Here we have again used the sign convention mentioned above; the fact that the map $\Xi \circ \mathscr{I}$ is of degree 0 ; and the fact that $\Xi\left(x \otimes p_{0}\right)=\xi$ and $\Xi\left(x \otimes p_{1}\right)=\eta$. This proves the identity.

We will also need the following:

Fact 2. $\partial\left\{(\Xi \circ \mathscr{I}) \cap^{*}\right\}=(\Xi \circ \mathscr{I}) \cap \partial^{*}+\xi \cap^{*}-\eta \cap^{*}-\eta \cup(\Xi \circ \mathscr{I}) \cap^{*}+$ $(\Xi \circ \mathscr{I}) \cup \xi \cap^{*}$.

Proof of Fact 2.

$$
\begin{aligned}
\partial\left\{(\boldsymbol{\Xi} \circ \mathscr{I}) \cap^{*}\right\} & =\partial(\boldsymbol{\Xi} \circ \mathscr{I}) \cap^{*}+(\boldsymbol{\Xi} \circ \mathscr{I}) \cap \partial^{*} \\
& =(\partial \Xi) \circ \mathscr{I} \cap^{*}-(\Xi \circ \partial \mathscr{I}) \cap^{*}+(\boldsymbol{\Xi} \circ \mathscr{I}) \cap \partial^{*} \\
& =-(\boldsymbol{\Xi} \cup \boldsymbol{\Xi}) \circ \mathscr{I} \cap^{*}-(\boldsymbol{\Xi} \circ \partial \mathscr{I}) \cap^{*}+(\boldsymbol{\Xi} \circ \mathscr{I}) \cap \partial^{*}
\end{aligned}
$$

(since $\Xi$ is a twisting cochain and, consequently, satisfies the identity $\partial \Xi+\Xi \cup \Xi=0)=-\eta \cup(\Xi \cdot \mathscr{I}) \cap^{*}+(\Xi \cdot \mathscr{I}) \cup \xi \cap^{*}-(\Xi \cdot \partial \mathscr{I}) \cap^{*}+(\Xi \circ \mathscr{I}) \cap \partial^{*}$. The remainder of the proof consists in observing that $(\partial \mathscr{I})(x)=$ $x \otimes p_{1}-x \otimes p_{0}$.

The remainder of the proof of the proposition follows by direct computation-i.e. let $x \otimes f \in X \otimes_{\xi} F$. Then

$$
\partial_{\xi}(x \otimes f)=\partial(x \otimes f)+\xi \cap(x \otimes f)
$$


and the image of this under $v$ is

$$
\partial(x \otimes f)+\xi \cap(x \otimes f)+(\Xi \cdot \mathscr{I}) \cap \partial(x \otimes f)+(\Xi \circ \mathscr{I}) \cup \xi \cap(x \otimes f) .
$$

Fact 2 above implies that this is equal to

$$
\begin{aligned}
\partial(x \otimes f) & +\xi \cap(x \otimes f)+(\Xi \circ \mathscr{I}) \cap \partial(x \otimes f)+(\Xi \circ \mathscr{I}) \cup \xi \cap(x \otimes f) \\
& +\partial\{(\Xi \circ \mathscr{I}) \cap(x \otimes f)\}-(\Xi \circ \mathscr{I}) \cap \partial(x \otimes f) \\
& -\xi \cap(x \otimes f)+\eta \cap(x \otimes f) \\
& +\eta \cup(\Xi \circ \mathscr{I}) \cap(x \otimes f)-(\Xi \circ \mathscr{I}) \cup \xi \cap(x \otimes f) \\
= & \partial(x \otimes f)+\partial\{(\Xi \circ \mathscr{I}) \cap(x \otimes f)\} \\
& +\eta \cap(x \otimes f)+\eta \cup(\Xi \circ \mathscr{I}) \cap(x \otimes f),
\end{aligned}
$$

which is the result of taking $\partial_{\eta}(x \otimes f+(\Xi \circ \mathscr{I}) \cap(x \otimes f))$.

Corollary B.14. Let $C$ be a commutative DGA-coalgebra, let $M$ be $a \mathbb{Q} \pi$-module and let $f_{i}: C \rightarrow \Sigma^{n+1} M_{*}$ be two chain-maps that are chain-homotopic via a chain-homotopy $h: C \rightarrow \Sigma^{n} M_{*}$. Then there exists an isomorphism of DGA-coalgebras $z: C \otimes_{t\left(f_{1}\right)} \mathfrak{U}(M, n, Q) \rightarrow C \otimes_{t\left(f_{2}\right)}$ $\mathfrak{U}(M, n, Q)$ with the following properties:

(a) $z$ commutes with the projections to $C$;

(b) $z$ is a homomorphism of $\mathfrak{U}(M, n, Q)$-modules.

Remarks. This implies that we can define two twisted tensor products to be equivalent, in this setting, if there exists a map satisfying the conclusions of the theorem. It is not difficult to show that, if two twisted tensor products are equivalent in this sense, their defining maps are chain-homotopic.

Proof. We define $\Xi$ by setting $\Xi \circ \mathscr{I}=e^{-h}-\eta=-h+h^{2} / 2 !-\ldots 7$ where $\eta: C \rightarrow \mathbb{Q} \subset \mathfrak{U}(M, n, Q)$ is the augmentation. This infinite series is perfectly well defined, when evaluated on any element of $C$-all but a finite number of terms vanish identically. This cochain has the property that $d \mathfrak{R}=-(d h) \cup \mathfrak{R}=-\left(t\left(f_{1}\right)-t\left(f_{2}\right)\right) \cup \mathfrak{R}$-this is the combinatorial property of "exponential" functions with respect to "differentiation". It, consequently defines a twisting cochain on $C \otimes I$ as in B.13.

Corollary B.15. Let $C$ be a commutative DGA-coalgebra, let $M$ be a $\mathbb{Q} \pi$-module and let $f: C \rightarrow \Sigma^{n+1} M_{*}$ be a chain map. Then $C \otimes_{t(f)}$ $\mathfrak{U}(M, n, Q)$ is determined, up to an isomorphism of DGA-coalgebras, by

\footnotetext{
${ }^{7}$ Exponentiation is done using cup-products.
} 
the class $\alpha(f) \in H^{n+1}(C, M)$, where $\alpha(f)$ is the composite $f: C_{n+1} \rightarrow$ $M_{0} \rightarrow M$.

REMARK. It is well known that the chain-homotopy class of a map to a resolution is determined by the cohomology class defined as above.

We conclude this appendix with some results on Eilenberg-Mac Lane spaces and algebraic models for them.

Proposition B.16. There exists a natural map of DGA-Hopf algebras $g: U(M, n) \rightarrow A(M, n)$ that induces a monomorphism in homology, in general, and an isomoprhism in rational homology.

Remarks. 1. This is proved in [7, $\S \S 17-19]$. When $n$ is even the map carries $\gamma_{t}(m) \in U(M, n)_{n t}$ to $\left[\left.\left.m\right|_{n} \cdots\right|_{n} m\right](t$ copies of $m) \in$ $A(M, n)_{n t}$. When $n$ is odd the map carries $m_{1} \wedge \cdots \wedge m_{t}$ to $\left[m_{1}\right] *$ $\cdots *\left[m_{t}\right]$ (shuffle product) $\in A(M, n)_{n t}$. The map given here is not an abelian group homomorphism on the chain level, but it induces a homomorphism of DGA algebras from $U(M, n)$ to $H_{*}(A(M, n))$. It fails to be a homomorphism on the chain level because the relation $\gamma_{t}(r m)=r^{t} \gamma_{t}(m)$ is satisfied in $U(M, n)$ but the corresponding relation isn't satisfied on the chain level in $A(M, n)$. The map to homology preserves coproducts.

2. It is possible to explicitly give a rational inverse to this map-i.e. a map $f$ from $A(M, n) \otimes \mathbb{Q} \rightarrow U(M, n) \otimes \mathbb{Q}$ that is well defined on the chain-level. Define:

$f\left(\left[\left.\left.m_{1}\right|_{\alpha} \cdots\right|_{\zeta} m_{p}\right]\right)=0$ if any of the subscripts of the bars, $\alpha \cdots \zeta$, are not equal to $n$;

$f\left(\left[\left.\left.m_{1}\right|_{n} \cdots\right|_{n} m_{t}\right]\right)=\gamma_{1}\left(m_{1}\right) \cdot \cdots \cdot \gamma_{1}\left(m_{t}\right) / t$ !, if $n$ is even, and;

$f\left(\left[\left.\left.m_{1}\right|_{n} \cdots\right|_{n} m_{t}\right]\right)=m_{1} \wedge \cdots \wedge m_{t} / t$ !, if $n$ is odd.

Proposition B.17. The map $f$ defined above induces isomorphisms in homology and preserves products.

Proof. We will first prove that it induces homology isomorphisms. Note that $f\left(\left[\left.\left.m\right|_{n} \cdots\right|_{n} m\right]\right)(t$ times $)=\gamma_{1}(m)^{t} / t !=\gamma_{t}(m)$ This implies that $f$ is surjective. If we can show that the boundary subgroup of $A(M, n)_{n t}$ is in the kernel we will be done, because that will imply that $f$ induces a well defined map of homology to $U(M, n)$. The conclusion will then follow from the fact that the composite with $g$ defined above is the identity map of $U(M, n)$. Let $a \in A(M, n)_{n t+1}$ be some element-we will show that $f$ (boundary of $a)=0$. This 
is clear if the boundary of $a$ has any $\left.\right|_{\alpha}$ in it with $\alpha \neq n$. We will, consequently, assume that boundary of $a$ has at least one term all of whose bars are of order $n$. This implies that $a$ has precisely $t$ bars of order $n$ and, at most, one of order 1-see the definition of $d_{T}$ above in B.16. (All terms of $d_{S}$ have bars of order $n-1$-so that component of the boundary can be ignored.) That implies that the boundary is of the form

$$
\begin{aligned}
& {\left[\left.\left.m_{1}\right|_{n} \cdots\right|_{n} m_{i}+\left.\left.m_{i^{\prime}}\right|_{n} \cdots\right|_{n} m_{t}\right]} \\
& -\left[\left.\left.m_{1}\right|_{n} \cdots\right|_{n} m_{i}|n \cdots|_{n} m_{t}\right]-\left[\left.\left.m_{1}\right|_{n} \cdots\left|{ }_{n} m_{i^{\prime}}\right| n \cdots\right|_{n} m_{t}\right] .
\end{aligned}
$$

This clearly maps to 0 under $f$.

The fact that $f$ is a DGA-algebra homomorphism now follows from the fact that it preserves products on elements of the form $\left[\left.\left.m\right|_{n} \cdots\right|_{n} m\right]$ and the fact that all other elements with all bars of order $n$ are equivalent to such elements modulo boundaries (which vanish under $f$ ).

This leads to one of the main results of this section:

THEOREM B.18. Let $Q$ be a projective $\mathbb{Q} \pi$-resolution of $\mathbb{Q}$ and suppose $M$ is a $\mathbb{Z}$-free $\mathbb{Z} \pi$-module, or a $\mathbb{Q} \pi$-module. Then there exists a chain-homotopy equivalence of DGA-algebras over $\mathbb{Q} \pi$

$$
f \otimes 1: A(M, n) \otimes Q \rightarrow U(M, n) \otimes Q .
$$

REMARKS. 1. This follows immediately from the fact that both terms are $\mathbb{Q} \pi$-projective after taking the tensor product with $Q$ and the fact that $f \otimes 1$ induces isomorphisms in homology.

2. This implies immediately that all of the homological $k$-invariants of $A(M, n) \otimes Q$ vanish identically.

Let $K(M, n)$ denote the DGA-coalgebra constructed by Quillen's theory for an Eilenberg-Mac Lane space. We will construct a DGAcoalgebra homomorphism from $K(M, n)$ to $U(M, n)$. We will need to recall some definitions due to Eilenberg and Mac Lane (see [6]):

Consider the cocycle, $c$, on $K(M, n)_{n}$ that maps $[m] \otimes 1 \otimes \cdots \otimes 1$ to $m \in M$, and let $\varepsilon: K(M, n) \rightarrow \mathbb{Z}$ be the augmentation. In addition, consider the infinite composite:

$\mathfrak{h}: K(M, n) \rightarrow \mathfrak{J}$, where $\mathfrak{h}=\cdots \cdot(\Delta \otimes 1 \otimes 1) \circ(\Delta \otimes 1) \circ \Delta, \Delta$ is the coproduct of $K(M, n)$, and $\mathfrak{J}$ is the inverse limit $K(M, n) \leftarrow K(M, n) \otimes K(M, n) \leftarrow$ $K(M, n) \otimes \cdots$-here we use the augmentation on the rightmost factor to project the tensor products. That there exists a well-defined map to the inverse limit, at all, follows from the fact that $(1 \otimes \varepsilon) \circ \Delta=1$. Now 
form the composite with $j_{k}=c^{k} \otimes \varepsilon \otimes \cdots: \mathfrak{J} \rightarrow M^{k}$, where the tensor product of maps has $k$ factors equal to $c$ and an infinite number of factors of $\varepsilon$ and the traget is a $\mathbb{Q}$-tensor product of $k$ copies of the $\mathbb{Q} \pi$-module $M$. Here we are regarding $M$ as a (nonprojective) $\mathbb{Z} \pi$ chain complex concentrated in dimension $n$. Note that the fact that $1 \otimes \varepsilon \cdot \Delta=1$ implies that $j_{k} \cdot \mathfrak{h}$ is the same as the composite of $j_{k}$ with a finite composite of $\Delta$ 's - call this $\mathfrak{h}_{k}$ in the remainder of this proof. We, consequently, get a sequence of chain-maps

$$
\mathfrak{s}_{k}=j_{k} \circ \mathfrak{h} / k !=j_{k} \circ \mathfrak{h}_{k} / k !: K(M, n) \rightarrow M^{k} .
$$

Now let $\mathfrak{m}: M_{i} \rightarrow U(M, n)_{i n}$ be the map that sends $m_{1} \otimes \cdots \otimes m_{i}$ to $m_{1} \cdots m_{i}$ utilizing the DGA-product structure of $U(M, n)$ and sends $1 \in \mathbb{Z}=M^{0}=U(M, n)_{0}$ to $1 \in U(M, n)_{0}$. The composite $\mathfrak{S}=\mathfrak{m} \circ\left(\varepsilon+\mathfrak{s}_{1}+\mathfrak{s}_{2}+\cdots\right): K(M, n) \rightarrow U(M, n)$ is also a chain-map. The main result of this section is the following:

THEOREM B.19. The map S: $K(M, n) \rightarrow U(M, n)$, defined above, induces isomorphisms in homology and is a homomorphism of DGAalgebras and of DGA-coalgebras.

Remarks. 1. Note that the statement about the map being a homomorphism of DGA-algebras implies that it preserves the product structures.

2. Note the factor of $k$ ! in the definition of $\mathfrak{s}_{k}$. This turns out to be crucial in the proof that $\mathfrak{S}$ preserves products.

Proof. 1. $\mathfrak{S}$ induces homology isomorphisms: This is an immediate consequence of the existence of the maps $g: A(M, n) \rightarrow K(M, n)$ and $f: A(M, n) \rightarrow U(M, n)$ defined, respectively, in [6] and the present paper. The first map is a chain-homotopy equivalence (see [16] where an explicit inverse is constructed) and preserves products and coproducts. The second map preserves products and coproducts, and consequently gives rise to a well-defined map in cohomology that preserves cupproducts. The complex $U(M, n)$ has the property that there exists a class $l \in H^{n}(U(M, n), M)$ evaluating to the identity map of homology, and with the property that its cup-products with itself also evaluate to isomorphisms in homology. The naturality of the evaluation map of cohomology on homology implies that $f^{*}(l) \in H^{n}(A(M, n), M)$ has this property as well. This immediately implies that $S$ induces homology isomorphisms because the cocycle $c$ is precisely a representative 
of the class that pulls back to $f^{*}(l)$ under $g$ and the construction of $\mathfrak{S}$ consists precisely in forming iterated cup-products of $c$ with itself and evaluating the result on homology.

2. $\mathfrak{S}$ preserves products and coproducts. This follows from the fact that $\mathfrak{S}$ is a map from the chain-complex to its homology whose composite with the map $U(M, n) \rightarrow A(M, n) \rightarrow K(M, n)$ is the identity. Here the leftmost map is the one mentioned in B.16 and the second map was defined by Eilenberg and Mac Lane in [6].

Appendix C-Proof of 2.10. We will use an inductive argument. In each step we will start with a homotopy-commutative diagram (let $H: T_{1} \rightarrow\left(T_{2}\right)_{n-1}$ be the homotopy):

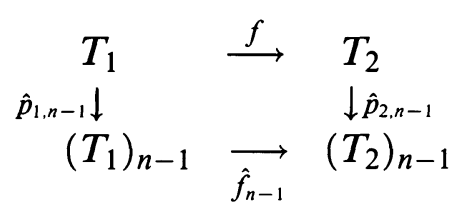

Figure C. 1

and the inductive step consists in constructing the corresponding $n$ thlevel diagram. The maps $\hat{p}_{i, j}, i=1,2$, are essentially like the projections of a $\mathbb{Q}$-model to its $j$ th stage-they are actually composites of these projections with DGA-coalgebra isomorphisms (in the initial case they are just the projections to the base). The isomorphisms $\mathscr{I}_{i}$ are $\lim \hat{p}_{i, j}$. In the induction step we:

1. form the universal contractible twisted tensor product (as in 2.7) to get $\left(T_{1}\right)_{n-1} \otimes_{x_{1}} \Omega\left(\left(T_{1}\right)_{n-1}\right)$ and $\left(T_{2}\right)_{n-1} \otimes_{x_{2}} \Omega\left(\left(T_{2}\right)_{n-1}\right)$. We pull these back over the maps $\hat{p}_{i, j}$ and an argument like that used in B.13 implies that $k_{n-1}=f \otimes \Omega\left(\hat{f}_{n-1}\right)+J \cap^{*}: T_{1} \otimes_{\hat{p}_{l, j} \cdot x_{1}} \Omega\left(\left(T_{1}\right)_{n-1}\right) \rightarrow$ $T_{2} \otimes_{\hat{p}_{2, j} \cdot x_{2}} \Omega\left(\left(T_{2}\right)_{n-1}\right)$ is a chain-map and even a homomorphism of DGA-coalgebras, where $J=e^{H}-1$, and exponents are formed with respect to cup-products. In addition the following diagram commutes:

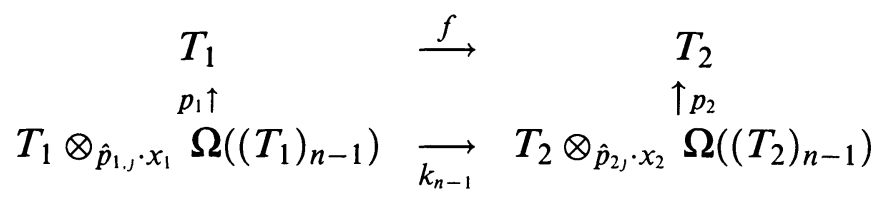

FIGURE C.2

where the $p_{i}$ are the projections of the twisted tensor products. 
2. Form the composites of the $p_{i}$ with the projections to the $n-1$ st stages to get the following diagram:

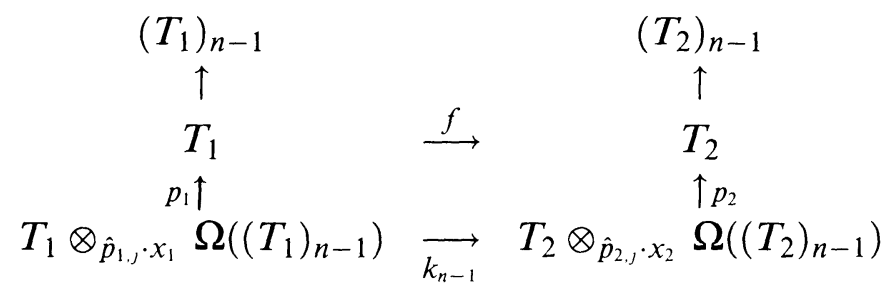

FIGURE C. 3

3. Note that the terms $T_{1} \otimes_{\hat{p}_{t}, x_{1}} \Omega\left(\left(T_{1}\right)_{n-1}\right)$ and $T_{2} \otimes_{\hat{p}_{2, j} \cdot x_{2}} \Omega\left(\left(T_{2}\right)_{n-1}\right)$ are acyclic below dimension $n$-in fact the projections $p_{i}, i=1,2$, are homotopic to the inclusions of the fibers when we regard the total space of the $\mathbb{Q}$-model as a twisted tensor product over the $(n-1)$ st stage. It follows that $H_{n}\left(T_{i} \otimes_{\hat{p}_{t, j} \cdot x_{i}} \Omega\left(\left(T_{i}\right)_{n-1}\right)\right)=\pi_{n}\left(T_{i}\right)$ and the pullback of the characteristic class of the $n$th stage of $T_{i}$ as a twisted tensor product over the $n-1^{\text {st }}$ stage will be a cocycle $\alpha_{i} \in H_{n}\left(T_{i} \otimes_{\hat{p}_{l, j} \cdot x_{i}} \Omega\left(\left(T_{i}\right)_{n-1}\right)\right)$ inducing an isomorphism in homology (upon evaluation).

4. We form the twisted tensor products of all the elements of the lower square of Figure C.3 over $T_{i}$ to get $D_{i, n-1}=\left(T_{i}\right)_{n-1} \otimes_{\tilde{x}_{t}}$ $\left(T_{i} \otimes_{\hat{p}_{l}, x_{i}} \Omega\left(\left(T_{i}\right)_{n-1}\right)\right)$-here $\tilde{x}_{i}:\left(T_{i}\right)_{n-1} \rightarrow T_{i} \otimes_{\hat{p}_{t}, \cdot x_{i}} \Omega\left(\left(T_{i}\right)_{n-1}\right)$ has its image entirely in $1 \otimes \Omega\left(\left(T_{i}\right)_{n-1}\right)$. The maps $\eta \otimes 1 \otimes \eta^{\prime}: D_{i, n-1} \rightarrow T_{i}$ are DGA-coalgebra homomorphisms that are also homology equivalences $-\eta$ and $\eta^{\prime}$ are the appropriate augmentation maps. Since $f_{n}^{*}\left(\alpha_{2}\right)$ is cohomologous to $\alpha_{1}$ the arguments of B.13 imply that

$$
\begin{aligned}
\hat{f}_{n-1} \otimes S\left(f_{n}^{*}\right) & +\Xi_{n} \cap^{*}:\left(T_{1}\right)_{n-1} \otimes_{\alpha_{1} \cdot \tilde{x}_{1}} \mathfrak{U}\left(\pi_{n}\left(T_{1}\right), n, Q\right) \\
& \rightarrow\left(T_{2}\right)_{n-1} \otimes_{\alpha_{2} \cdot \tilde{x}_{2}} \mathfrak{U}\left(\pi_{n}\left(T_{2}\right), n, Q\right)
\end{aligned}
$$

is a DGA-coalgebra homomorphism, where $\Xi_{n}=e^{\beta}$, and $\delta \beta=\alpha_{1}-$ $f_{n}^{*}\left(\alpha_{2}\right)$. The way the $\alpha_{i}$ were chosen implies that $\left(T_{i}\right)_{n-1} \otimes_{\alpha_{i} \cdot \tilde{x}_{t}}$ $\mathfrak{U}\left(\pi_{n}\left(T_{i}\right), n, Q\right)$ is isomorphic to $\left(T_{i}\right)_{n}$ and that the maps $\hat{p}_{i, n-1}: T_{i} \rightarrow$ $\left(T_{i}\right)_{n-1}$ extend to maps

$$
\hat{p}_{i, n}: T_{i} \rightarrow\left(T_{i}\right)_{n}=\left(T_{i}\right)_{n-1} \otimes_{\alpha_{l} \cdot \tilde{x}_{l}} \mathfrak{U}\left(\pi_{n}\left(T_{i}\right), n, Q\right),
$$

where the $n$th homotopy module of $T_{i}$ is essentially mapped via $\alpha_{i}$. If we define $\hat{f}_{n}=\hat{f}_{n-1} \otimes S\left(f_{n}^{*}\right)+\Xi_{n} \cap^{*}$ the result follows. 


\section{REFERENCES}

[1] H. Baues, On the homotopoy classification problem, preprint (Sonderforschungsbereich 40 'Theoretische Mathematik' Universität Bonn und Max-Planck Institut), 1983.

[2] H. Baues and L. Lemaire, Minimal models in homotopy theory, Math. Ann., 225 (1977), 219-242.

[3] E. Brown, Twisted tensor products, I, Ann. of Math., 69 (1959), 223-246.

[4] G. Carlsson, A counterexample to a conjecture of Steenrod, Invent. Math., 64 (1981), 171-174.

[5] H. Cartan, Algebras d'Eilenberg-MacLane et Homotopie, Seminaire Henri Car$\tan 1954 / 55$, ENS, Paris.

[6] S. Eilenberg and S. MacLane, On the groups $H(\Pi, n)$. I, Ann. of Math., 58 (1954), 55-106.

[7] On the groups $H(\Pi, n)$. II, Ann. of Math., 60 (1954), 49-139.

[8] V. K. A. M. Gugenheim, On a theorem of E. H. Brown, Illinois J. Math., 4 (1960), 292-311.

[9] On the chain-complex of a fibration, Illinois J. Math., 16 (1972), 398414.

[10] V. K. A. M. Gugenheim, M. G. Barrett, J. C. Moore, On semisimplicial fiberbundles, Amer. J. Math., 81 (1959), 639-657.

[11] D. Quillen, Rational homotopy theory, Annals of Math., 90 (1969), 205-295.

[12] Homotopical Algebra, Springer-Verlag Lecture Notes in Mathematics no. $43,1967$.

[13] M. Rothenberg and G. Triantafillou, An Algebraic Model for Simple Homotopy Types, U. of Minn. Math. Report 83-112.

[14] Shih Weishu, Homologie des espaces fibrés, I.H.E.S Publ. Math., 13 (1962), 93-176.

[15] J. Smith. Equivariant Moore Spaces, Springer-Verlag Lecture Notes in Mathematics, 1126 (1983), 238-270.

[16] _ Equivariant Moore spaces. II-The low-dimensional case, J. Pure Appl. Alg., 36 (1985), 187-204.

[17] _ Topological realizations of chain complexes. I-The general theory, Topology Appl., 22 (1986), 301-313.

[18] D. Sullivan, Infinitesimal computations in topology, I.H.E.S Publ. Math., 47 (1977), 269-331.

[19] C. T. C. Wall, Finiteness conditions for CW-complexes. II, Proc. Roy. Soc. A, 295 (1960), 129-139.

Received September 7, 1987.

DREXEL UNIVERSITY

Philadelphia, PA 19104 